\title{
Review Article \\ $U(3)_{C} \times S p(1)_{L} \times U(1)_{L} \times U(1)_{R}$
}

\section{Luis Alfredo Anchordoqui}

Department of Physics, University of Wisconsin-Milwaukee, P.O. Box 413, Milwaukee, WI 53201, USA

Correspondence should be addressed to Luis Alfredo Anchordoqui, anchordo@uwm.edu

Received 30 August 2011; Revised 17 October 2011; Accepted 20 October 2011

Academic Editor: Ira Rothstein

Copyright (C) 2012 Luis Alfredo Anchordoqui. This is an open access article distributed under the Creative Commons Attribution License, which permits unrestricted use, distribution, and reproduction in any medium, provided the original work is properly cited.

We outline the basic setting of the $U(3)_{C} \times S p(1)_{L} \times U(1)_{L} \times U(1)_{R}$ gauge theory and review the associated phenomenological aspects related to experimental searches for new physics at hadron colliders. In this construction, there are two massive $Z^{\prime}$-gauge bosons, which can be naturally associated with baryon number $B$ and $B-L$ ( $L$ being lepton number). We discuss the potential signals which may be accessible at the Tevatron and at the Large Hadron Collider (LHC). In particular, we provide the relevant cross sections for the production of $Z^{\prime}$-gauge bosons in the TeV region, leading to predictions that are within reach of the present or the next LHC run. After that we direct attention to embedding the gauge theory into the framework of string theory. We consider extensions of the standard model based on open strings ending on D-branes, with gauge bosons due to strings attached to stacks of D-branes and chiral matter due to strings stretching between intersecting D-branes. Assuming that the fundamental string mass scale is in the $\mathrm{TeV}$ range and the theory is weakly coupled, we explore the LHC discovery potential for Regge excitations.

\section{General Idea}

The recent development in high energy physics has put a great emphasis on gauge theories; indeed the general theory of fundamental interactions, rather unimaginatively named the Standard Model (SM), is completely formulated in this framework. The SM agrees remarkably well with current data but has rather troubling weaknesses and appears to be a somewhat ad hoc theory. It is thought that the SM may be a subset of a more fundamental gauge theory. Several models have been explored, using the fundamental principle of gauge invariance as guidepost. The purpose of this paper is to outline the main phenomenological aspects of one such models: $U(3)_{C} \times S p(1)_{L} \times U(1)_{L} \times U(1)_{R}$. The first aim is to survey the basic features of the gauge theory's prediction regarding the new mass sector and couplings. These features lead to new phenomena that can be probed using data from the Tevatron and the Large Hadron Collider (LHC). In particular the theory predicts that additional gauge bosons 
that we will show are accessible at LHC energies. Having so identified the general properties of the theory, we focus on the potential to embed this model into a string theory. We show this can be accomplished within the context of D-brane TeV-scale compactifications. Finally, we explore predictions inherited from properties of the overarching string theory.

The SM is a spontaneously broken Yang-Mills theory with gauge group $S U(3)_{C} \times$ $S U(2)_{L} \times U(1)_{Y}$. Matter in the form of quarks and leptons (i.e., $S U(3)_{C}$ triplets and singlets, resp.) is arranged in three families $(i=1,2,3)$ of left-handed fermion doublets (of $\left.S U(2)_{L}\right)$ and right-handed fermion singlets. Each family $i$ contains chiral gauge representations of left-handed quarks $Q_{i}=(3,2)_{1 / 6}$ and leptons $L_{i}=(1,2)_{-1 / 2}$ as well as right-handed up and down quarks, $U_{i}=(3,1)_{2 / 3}$ and $D_{i}=(3,1)_{-1 / 3}$, respectively, and the right-handed lepton $E_{i}=(1,1)_{-1}$. The hypercharge $Y$ is shown as a subscript of the $S U(3)_{C} \times S U(2)_{L}$ gauge representation $(A, B)$. The neutrino is part of the left-handed lepton representation $L_{i}$ and does not have a right-handed counterpart.

The SM Lagrangian exhibits an accidental global symmetry $U(1)_{B} \times U(1)_{e} \times U(1)_{\mu} \times$ $U(1)_{\tau}$, where $U(1)_{B}$ is the baryon number symmetry and $U(1)_{\alpha}(\alpha=e, \mu, \tau)$ are three lepton flavor symmetries, with total lepton number given by $L=L_{e}+L_{\mu}+L_{\tau}$. It is an accidental symmetry because we do not impose it. It is a consequence of the gauge symmetries and the low energy particle content. It is possible (but not necessary), however, that effective interaction operators induced by the high energy content of the underlying theory may violate sectors of the global symmetry.

The electroweak subgroup $S U_{L}(2) \times U_{Y}(1)$ is spontaneously broken to the electromagnetic $U(1)_{\mathrm{EM}}$ by the Higgs doublet $H=(1,2)_{1 / 2}$ which receives a vacuum expectation value $v \neq 0$ in a suitable potential. Three of the four components of the complex Higgs are "eaten" by the $W^{ \pm}$and $Z$ bosons, which are superpositions of the gauge bosons $W_{\mu}^{a}$ of $S U(2)_{L}$ and $B_{\mu}$ of $U(1)_{Y}$,

$$
\begin{gathered}
W_{\mu}^{ \pm}=\frac{1}{\sqrt{2}} W_{\mu}^{1} \mp \frac{i}{\sqrt{2}} W_{\mu}^{2} \\
Z_{\mu}=\cos \theta_{W} W_{\mu}^{3}-\sin \theta_{W} B_{\mu}
\end{gathered}
$$

with masses $M_{W}^{2}=\pi \alpha v^{2} / \sin ^{2} \theta_{W}, M_{Z}^{2}=M_{W}^{2} / \cos ^{2} \theta_{W}$, and $\alpha \simeq 1 / 128$ at $Q^{2}=M_{W}^{2}$. The fourth vector field,

$$
A_{\mu}=\sin \theta_{W} W_{\mu}^{3}+\cos \theta_{W} B_{\mu}
$$

persists massless, and the remaining Higgs component is left as a $U(1)_{\mathrm{EM}}$ neutral real scalar. The measured values $M_{W} \simeq 80.4 \mathrm{GeV}$ and $M_{Z} \simeq 91.2 \mathrm{GeV}$ fix the weak mixing angle at $\sin ^{2} \theta_{W} \simeq 0.23$ and the Higgs vacuum expectation value at $\langle H\rangle=v \simeq 246 \mathrm{GeV}$ [1].

Fermion masses arise from Yukawa interactions, which couple the right-handed fermion singlets to the left-handed fermion doublets and the Higgs field,

$$
\mathcal{L}=-Y_{d}^{i j} \bar{Q}_{i} H D_{j}-Y_{u}^{i j} \epsilon^{a b} \bar{Q}_{i a} H_{b}^{\dagger} U_{j}-Y_{\ell}^{i j} \bar{L}_{i} H E_{j}+\text { h.c., }
$$

where $\epsilon^{a b}$ is the antisymmetric tensor. In the process of spontaneous symmetry breaking, these interactions lead to charged fermion masses, $m_{f}^{i j}=Y_{f}^{i j} v / \sqrt{2}$, but leave the neutrinos 
massless [2]. (One might think that neutrino masses could arise from loop corrections. This, however, cannot be the case, because the only possible neutrino mass term that can be constructed with the SM fields is the bilinear $\bar{L}_{i} L_{j}^{C}$ which violates the total lepton symmetry by two units $\left(L_{i}^{C}=C \bar{L}_{i}^{T}\right)$. As mentioned above, total lepton number is a global symmetry of the model and therefore $L$-violating terms cannot be induced by loop corrections. Furthermore, the $U(1)_{B-L}$ subgroup is nonanomalous, and therefore $B-L$ violating terms cannot be induced even by nonperturbative corrections. It follows that the SM predicts that neutrinos are strictly massless.) Experimental evidence for neutrino flavor oscillations by the mixing of different mass eigenstates implies that the SM has to be extended [3]. The most economic way to get massive neutrinos would be to introduce the right-handed neutrino states (having no gauge interactions, these sterile states would be essentially undetectable) and obtain a Dirac mass term through a Yukawa coupling.

The SM gauge interactions have been tested with unprecedented accuracy, including some observables beyond even one part in a million [1]. Nevertheless, the saga of the SM is still exhilarating because it leaves all questions of consequence unanswered. The most evident of unanswered questions is why there is a huge disparity between the strength of gravity and of the SM forces. This hierarchy problem suggests that new physics could be at play at the TeV-scale and is arguably the driving force behind high energy physics for several decades. Much of the motivation for anticipating the existence of such new physics is based on considerations of naturalness. The nonzero vacuum expectation value of the scalar Higgs doublet condensate sets the scale of electroweak interactions. However, due to the quadratic sensitivity of the Higgs mass to quantum corrections from an arbitrarily high mass scale $\Lambda$, with no new physics between the energy scale of electroweak unification and the vicinity of the Planck mass, the bare Higgs mass and quantum corrections have to cancel at a level of one part in $\sim 10^{30}$. This fine-tuned cancellation seems unnatural, even though it is in principle self-consistent. Thus either the scale of new physics $\Lambda$ is much smaller than the Planck scale or there exists a mechanism which ensures this cancellation, perhaps arising from a new symmetry principle beyond the SM; minimal supersymmetry (SUSY) is a textbook example [4]. In either case, an extension of the SM appears necessary.

In this paper we examine the phenomenology of a newfangled extension of the gauge sector, $U(3)_{C} \times S p(1)_{L} \times U(1)_{L} \times U(1)_{R}$, which has the attractive property of elevating the two major global symmetries of the SM $(B$ and $L)$ to local gauge symmetries [5]. (The fundamental principles of the model are summarized in [6-8]. Herein though we replace at full length the $U(2)_{L}$ doublets by $S p(1)_{L}$ doublets. Besides the fact that this reduces the number of extra $U(1)^{\prime} \mathrm{s}$, one avoids the presence of a problematic Peccei-Quinn symmetry [9-11], associated in general with the $U(1)$ of $U(2)_{L}$ under which Higgs doublets are charged [12]. A point worth noting at this juncture: the compact symplectic group $S p(1)$ is equivalent to $S U(2)$; our choice of notation will become clear in Section 5.) The $U(1)_{Y}$ boson $Y_{\mu}$, which gauges the usual electroweak hypercharge symmetry, is a linear combination of the $U(1)$ of $U(3)_{C}$ gauge boson $C_{\mu}$, the $U(1)_{R}$ boson $B_{\mu}$, and a third additional $U(1)_{L}$ field $\widetilde{B}_{\mu}$. The $Q_{3}$, $Q_{1 L}, Q_{1 R}$ content of the hypercharge operator is given by

$$
Q_{Y}=c_{1} Q_{1 R}+c_{3} Q_{3}+c_{4} Q_{1 L}
$$

with $c_{1}=1 / 2, c_{3}=1 / 6$, and $c_{4}=-1 / 2$ [13]. The corresponding fermion and Higgs doublet quantum numbers are given in Table 1 . The criteria we adopt here to define the Higgs charges are to make the Yukawa couplings $\left(H \bar{U}_{i} Q_{i}, H^{\dagger} \bar{D}_{i} Q_{i}, H^{\dagger} \bar{E}_{i} L_{i}, H \bar{N}_{i} L_{i}\right)$ invariant under all 
Table 1: Quantum numbers of chiral fermions and Higgs doublet.

\begin{tabular}{lccccc}
\hline Name & Representation & $Q_{3}$ & $Q_{1 L}$ & $Q_{1 R}$ & $Q_{Y}$ \\
\hline$Q_{i}$ & $(3,2)$ & 1 & 0 & 0 & $\frac{1}{6}$ \\
$\bar{U}_{i}$ & $(\overline{3}, 1)$ & -1 & 0 & -1 & $-\frac{2}{3}$ \\
$\bar{D}_{i}$ & $(\overline{3}, 1)$ & -1 & 0 & 1 & $\frac{1}{3}$ \\
$L_{i}$ & $(1,2)$ & 0 & 1 & 0 & $-\frac{1}{2}$ \\
$\bar{E}_{i}$ & $(1,1)$ & 0 & -1 & 1 & 1 \\
$\bar{N}_{i}$ & $(1,1)$ & 0 & -1 & -1 & 0 \\
$H$ & $(1,2)$ & 0 & 0 & 1 & $\frac{1}{2}$ \\
\hline
\end{tabular}

three $U(1)$ 's. From Table $1, \bar{U}_{i} Q_{i}$ has the charges $(0,0,-1)$ and $\bar{D}_{i} Q_{i}$ has $(0,0,1)$; therefore, the Higgs $H$ has $Q_{3}=Q_{1 L}=0, Q_{1 R}=1, Q_{Y}=1 / 2$, whereas $H^{\dagger}$ has opposite charges $Q_{3}=Q_{1 L}=0, Q_{1 R}=-1, Q_{Y}=-1 / 2$. The two extra $U(1)$ 's are the baryon and lepton number; they are given by the following combinations:

$$
B=\frac{Q_{3}}{3}, \quad L=Q_{1 L}, \quad Q_{Y}=\frac{1}{6} Q_{3}-\frac{1}{2} Q_{1 L}+\frac{1}{2} Q_{1 R},
$$

or equivalently by the inverse relations

$$
Q_{3}=3 B, \quad Q_{1 L}=L, \quad Q_{1 R}=2 Q_{Y}-(B-L) .
$$

Even though $B$ is anomalous, with the addition of three fermion singlets $N_{i}$, the combination $B-L$ is anomaly free. One can verify by inspection of Table 1 that these $N_{i}$ have the quantum numbers of right-handed neutrinos, that is, singlets under hypercharge. Therefore, this is a first interesting prediction of the $U(3)_{C} \times S p(1)_{L} \times U(1)_{L} \times U(1)_{R}$ gauge theory: right-handed neutrinos must exist.

Before discussing the favorable phenomenological implications of the model, we detail some desirable properties which apply to generic models with multiple $U(1)$ symmetries.

\section{Running of the Abelian Gauge Couplings}

We begin with the covariant derivative for the $U(1)$ fields in the "flavor" $1,2,3, \ldots$ basis in which it is assumed that the kinetic energy terms containing $X_{\mu}^{i}$ are canonically normalized:

$$
\Phi_{\mu}=\partial_{\mu}-i \sum g_{i}^{\prime} Q_{i} X_{\mu}^{i} .
$$


The relations between the $U(1)$ couplings $g_{i}^{\prime}$ and any nonabelian counterparts are left open for now. We carry out an orthogonal transformation of the fields $X_{\mu}^{i}=\sum_{j} O_{i j} Y_{\mu}^{j}$. The covariant derivative becomes

$$
\begin{aligned}
\Phi_{\mu} & =\partial_{\mu}-i \sum_{i} \sum_{j} g_{i}^{\prime} Q_{i} O_{i j} Y_{\mu}^{j} \\
& =\partial_{\mu}-i \sum_{j} \bar{g}_{j} \bar{Q}_{j} Y_{\mu \prime}^{j}
\end{aligned}
$$

where for each $j$

$$
\bar{g}_{j} \bar{Q}_{j}=\sum_{i} g_{i}^{\prime} Q_{i} O_{i j}
$$

Next, suppose we are provided with normalization for the hypercharge (taken as $j=1$ )

$$
Q_{Y}=\sum_{i} c_{i} Q_{i}
$$

hereafter we omit the bars for simplicity. Rewriting (2.3) for the hypercharge

$$
g_{Y} Q_{Y}=\sum_{i} g_{i}^{\prime} Q_{i} O_{i 1}
$$

and substituting (2.4) into (2.5), we obtain

$$
g_{\Upsilon} \sum_{i} Q_{i} c_{i}=\sum_{i} g_{i}^{\prime} O_{i 1} Q_{i}
$$

One can think about the charges $Q_{i, p}$ as vectors with the components labeled by particles $p$. Let us first take the charges to be orthogonal, that is, $\sum_{p} Q_{i, p} Q_{k, p}=0$ for $i \neq k$. Multiplying (2.6) by $\sum_{p} Q_{k, p}$,

$$
\sum_{p} Q_{k, p} g_{Y} \sum_{i} Q_{i, p} c_{i}=\sum_{p} Q_{k, p} \sum_{i} g_{i}^{\prime} O_{i 1} Q_{i, p}
$$

we obtain

$$
g_{Y} c_{i}=g_{i}^{\prime} O_{i 1},
$$

or equivalently

$$
O_{i 1}=\frac{g_{Y} c_{i}}{g_{i}^{\prime}}
$$


Orthogonality of the rotation matrix, $\sum_{i} O_{i 1}^{2}=1$, implies

$$
g_{Y}^{2} \sum_{i}\left(\frac{c_{i}}{g_{i}^{\prime}}\right)^{2}=1
$$

Then, the condition

$$
P \equiv \frac{1}{g_{Y}^{2}}-\sum_{i}\left(\frac{c_{i}}{g_{i}^{\prime}}\right)^{2}=0
$$

encodes the orthogonality of the mixing matrix connecting the fields coupled to the flavor charges $Q_{1}, Q_{2}, Q_{3}, \ldots$ and the fields rotated, so that one of them, $Y$, couples to the hypercharge $Q_{Y}$. Therefore, for orthogonal charges, as the couplings run with energy, the condition $P=0$ needs to stay intact [5].

A very important point is that the couplings that are running are those of the $U(1)$ fields; hence the $\beta$ functions receive contributions from fermions and scalars, but not from gauge bosons. As a consequence, if we start with a set of couplings at a high mass scale $\Lambda$ satisfying $P=0$, this condition will be maintained at one loop as the couplings run down to lower energies $(Q)$. The one-loop correction to the various couplings is

$$
\begin{gathered}
\frac{1}{\alpha_{Y}(Q)}=\frac{1}{\alpha_{Y}(\Lambda)}-\frac{b_{Y}}{2 \pi} \ln \left(\frac{Q}{\Lambda}\right), \\
\frac{1}{\alpha_{i}(Q)}=\frac{1}{\alpha_{i}(\Lambda)}-\frac{b_{i}}{2 \pi} \ln \left(\frac{Q}{\Lambda}\right),
\end{gathered}
$$

where

$$
\begin{aligned}
b_{Y} & =\frac{2}{3} \operatorname{Tr} Q_{Y, f}^{2}+\frac{1}{3} \operatorname{Tr} Q_{Y, S^{\prime}}^{2} \\
b_{i} & =\frac{2}{3} \operatorname{Tr} Q_{i, f}^{2}+\frac{1}{3} \operatorname{Tr} Q_{i, S^{\prime}}^{2}
\end{aligned}
$$

with $f$ and $s$ indicating contribution from fermion and scalar loops, respectively.

Recall that the charges are orthogonal, $\sum_{s} Q_{i, s} Q_{k, s}=\sum_{f} Q_{i, f} Q_{k, f}=0$ for $i \neq k$. Then (2.4) implies

$$
\sum_{s} Q_{Y, s}^{2}=\sum_{i} c_{i}^{2} \sum_{s} Q_{i, s^{\prime}}^{2} \quad \sum_{f} Q_{Y, f}^{2}=\sum_{i} c_{i}^{2} \sum_{f} Q_{i, f}^{2}
$$

hence,

$$
b_{Y}=\sum_{i} c_{i}^{2} b_{i}
$$


On the other, the RG-induced change of $P$ defined in (2.11) reads

$$
\begin{aligned}
\Delta P & =\Delta\left(\frac{1}{\alpha_{Y}}\right)-\sum_{i} c_{i}^{2} \Delta\left(\frac{1}{\alpha_{i}}\right) \\
& =\frac{1}{2 \pi}\left(b_{Y}-\sum_{i} c_{i}^{2} b_{i}\right) \ln \left(\frac{Q}{\Lambda}\right) .
\end{aligned}
$$

Thus, $P=0$ stays valid to one loop if the charges are orthogonal [5].

Should the charges not be orthogonal, it is instructive to write (2.6) as $\mathbb{V} \cdot \mathbb{Q}=0$, where

$$
V_{i}=O_{i 1}-\frac{g_{\gamma} c_{i}}{g_{i}^{\prime}}
$$

Certainly $V_{i}=0$ still holds as a possible solution. But as the charges do not form a mutually orthogonal basis, one can ask whether other solutions exist. This will be the case if, for nonzero $\mathbb{V}$,

$$
\sum_{i} V_{i} Q_{i}^{\alpha}=0
$$

for each $\alpha$, where $Q_{i}^{\alpha}$ is the $U(1)$ charge of the particle $\alpha$. In the $U(3) \times U(2) \times U(1)$ gauge group of [12], the right-handed electron is charged only with respect to one of the abelian groups. From (2.19), this sets one of the $V^{\prime}$ s (say $V_{1}$ ) equal to zero. For $\alpha=Q_{i}, U_{i}$, $D_{i}, L_{i}, E_{i}, N_{i}, H$, there remain at least 4 additional equations satisfied by the remaining components $V_{2}$ and $V_{3}$. The resulting overcompleteness leads to $V_{2}=V_{3}=0$.

Although in most models the condition $P=0$ holds in spite of the nonorthogonality of the $Q_{i}$ 's, the RG equations controlling the running of the couplings lose their simplicity. In particular, since

$$
\operatorname{Tr} Q_{Y}^{2} \neq \sum_{i} c_{i}^{2} \operatorname{Tr} Q_{i}^{2}
$$

the RG equations become coupled. In addition, kinetic mixing is generated at one loop level even if it is absent initially [14,15]. Removal of the mixing term in order to restore canonical gauge kinetic energy requires an additional $O(3)$ rotation, greatly complicating the analysis.

Here, we are considering models where the underlying symmetry at high energies is $U(N)$ rather than $S U(N)$. Following [12], we normalize all $U(N)$ generators according to

$$
\operatorname{Tr}\left(T^{a} T^{b}\right)=\frac{1}{2} \delta^{a b}
$$

and measure the corresponding $U(1)_{N}$ charges with respect to the coupling $g_{N} / \sqrt{2 N}$, with $g_{N}$ as the $S U(N)$ coupling constant. Hence, the fundamental representation of $S U(N)$ has $U(1)_{N}$ charge unity. Another important element of the RG analysis is that the $U(1)$ couplings $\left(g_{1}^{\prime}, g_{2}^{\prime}, g_{3}^{\prime}\right)$ run different from the nonabelian $S U(3)\left(g_{3}\right)$ and $S U(2)\left(g_{2}\right)$. This implies that 
the previous relation for normalization of abelian and nonabelian coupling constants, $g_{N}^{\prime}=$ $g_{N} / \sqrt{2 N}$, holds only at the scale of $U(N)$ unification [5]. The SM chiral fermion charges in Table 1 are not orthogonal as given $\left(\operatorname{Tr} Q_{1 L} Q_{1 R} \neq 0\right.$,). Orthogonality can be completed by including a right-handed neutrino.

An obvious question is whether each of the fields on the rotated basis couples to a single charge $\bar{Q}_{i}$. Let

$$
\mathcal{L}=\mathbb{X}^{T} \mathbb{G} \mathbb{Q}
$$

be the Lagrangian in the $1,2,3, \ldots$ basis, with $X_{\mu}^{i}$ and $Q_{i}$ vectors and $\mathbb{G}$ a diagonal matrix in $N$-dimensional "flavor" space. Now rotate to new orthogonal basis $(\overline{\mathbb{Q}})$ for $\mathbb{Q}$ :

$$
\mathbb{Q}=\mathbb{R} \overline{\mathbb{Q}}
$$

equation (2.22) becomes

$$
\mathcal{L}=\mathbb{X}^{T} \mathbb{G} \mathbb{R} \overline{\mathbb{Q}}
$$

As it stands, each $X_{\mu}^{i}$ does not couple to a unique charge $\bar{Q}_{i}$; hence we rotate $\mathbb{X}$,

$$
\mathbb{X}=\mathbb{O} \bar{Y}
$$

to obtain

$$
\mathcal{L}=\overline{\mathbb{Y}}^{T} \mathbb{O}^{T} \mathbb{G} \mathbb{R} \overline{\mathbb{Q}}
$$

We wish to see if, for given $\mathbb{O}$ and $\mathbb{G}$, we can find an $\mathbb{R}$ so that

$$
\mathbb{O}^{T} \mathbb{G} R=\overline{\mathbb{G}} \text { (diagonal). }
$$

This allows each $\bar{Y}_{\mu}^{i}$ to couple to a unique charge $\bar{Q}_{i}$ with strength $\bar{g}_{i}$. To see the problem with this, we rewrite (2.27) in terms of components

$$
\left(O^{T}\right)_{i j} g_{j} R_{j k}=\bar{g}_{i} \delta_{i k}
$$

for $i \neq k,(2.28)$ leads to

$$
\left(O^{T}\right)_{i j} g_{j} R_{j k}=0
$$


In general, in (2.29) there are $N(N-1)$ equations, but only $N(N-1) / 2$ independent $O_{i j}$ generators in $S O(N)$; therefore the system is overdetermined [16]. Of course, if $\mathbb{G}=g \mathbb{I}$, the equation becomes

$$
\mathbb{O}^{T} \mathbb{R}=\mathbb{I}
$$

and so $\mathbb{O}=\mathbb{R}$.

We illustrate with the case $N=2$; let

$$
\begin{gathered}
\mathbb{R}=\left(\begin{array}{cc}
C_{\varphi} & S_{\varphi} \\
-S_{\varphi} & C_{\varphi}
\end{array}\right), \\
\mathbb{G}=\left(\begin{array}{cc}
g_{1}^{\prime} & 0 \\
0 & g_{3}^{\prime}
\end{array}\right), \\
\mathbb{O}=\left(\begin{array}{cc}
C_{\vartheta} & S_{\vartheta} \\
-S_{\vartheta} & C_{\vartheta}
\end{array}\right),
\end{gathered}
$$

then,

$$
\mathbb{O G R}=\left(\begin{array}{ll}
g_{1}^{\prime} C_{\vartheta} C_{\varphi}+g_{3}^{\prime} S_{\vartheta} S_{\varphi} & g_{1}^{\prime} C_{\vartheta} S_{\varphi}-g_{3}^{\prime} S_{\vartheta} C_{\varphi} \\
g_{1}^{\prime} S_{\vartheta} C_{\varphi}+g_{3}^{\prime} C_{\vartheta} S_{\varphi} & g_{1}^{\prime} S_{\vartheta} S_{\varphi}-g_{3}^{\prime} C_{\vartheta} C_{\varphi}
\end{array}\right)=\left(\begin{array}{cc}
\bar{g}_{1}^{\prime} & 0 \\
0 & \bar{g}_{3}^{\prime}
\end{array}\right)
$$

From the off-diagonal terms, we obtain

$$
\begin{aligned}
& g_{1}^{\prime} C_{\vartheta} S_{\varphi}-g_{2}^{\prime} S_{\vartheta} C_{\varphi}=0 \Longrightarrow \tan \vartheta=\frac{g_{1}^{\prime}}{g_{2}^{\prime}} \tan \varphi \\
& g_{1}^{\prime} S_{\vartheta} C_{\varphi}-g_{2}^{\prime} C_{\vartheta} S_{\varphi}=0 \Longrightarrow \tan \vartheta=\frac{g_{2}^{\prime}}{g_{1}^{\prime}} \tan \varphi
\end{aligned}
$$

which implies that $g_{1}^{\prime}=g_{2}^{\prime}=g$ or equivalently that $\mathbb{G}$ is a multiple of the unit matrix. Next, we consider the diagonal elements using $g_{1}^{\prime}=g_{2}^{\prime}$ to obtain

$$
\cos (\vartheta-\varphi)=0 \Longrightarrow \vartheta=\varphi
$$

Note that the matrix $\mathbb{R}$ has one independent variable, and there are two independent homogeneous equations.

Any vector boson $Y_{\mu}^{\prime}$, orthogonal to the hypercharge, must grow a mass $M^{\prime}$ in order to avoid long range forces between baryons other than gravity and Coulomb forces. The anomalous mass growth allows the survival of global baryon number conservation, preventing fast proton decay [17]. It is this that we now turn to study. 


\section{Premises of the Anomalous Sector}

Outside of the Higgs couplings, the relevant parts of the Lagrangian are the gauge couplings generated by the $U(1)$ covariant derivatives acting on the matter fields and the (mass) ${ }^{2}$ matrix of the anomalous sector

$$
\mathcal{L}=\mathbb{Q}^{T} \mathbb{G} \mathbb{X}+\frac{1}{2} \mathbb{X}^{T} \mathbb{M}^{2} \mathbb{X}
$$

where $X_{\mu}^{i}$ are the three $U(1)$ gauge fields in the D-brane basis $\left(B_{\mu}, C_{\mu}, \widetilde{B}_{\mu}\right), \mathbb{G}$ is a diagonal coupling matrix $\left(g_{1}^{\prime}, g_{3}^{\prime}, g_{4}^{\prime}\right)$, and $\mathbb{Q}$ are the 3 charge matrices.

Again, perform a rotation $\mathbb{X}=\mathbb{O} \mathbb{Y}$ and require that one of the $\mathbb{Y}^{\prime}$ 's (say $Y_{\mu}$ ) couples to hypercharge. We then obtain the constraint on the first column of $\mathbb{O}$ given in (2.9). However, there is now an additional constraint: the field $Y_{\mu}$ is an eigenstate of $\mathbb{M}^{2}$ with zero eigenvalue. Under the $\mathbb{O}$ rotation, the mass term becomes

$$
\frac{1}{2} \mathbb{X}^{T} \mathbb{M}^{2} \mathbb{X}=\frac{1}{2} \mathbb{Y}^{T} \overline{\mathbb{M}^{2}} \mathbb{Y}
$$

with $\overline{\mathbb{M}^{2}}=\mathbb{O}^{T} \mathbb{M}^{2} \mathbb{O}$. We know that at least $Y_{\mu}$ is an eigenstate with eigenvalue zero. We also know that Poincare invariance requires the complete diagonalization of the mass matrix in order to deal with observables. However, further similarity transformations will undo the coupling of the zero eigenstate to hypercharge. There seems no way of eventually fulfilling all these conditions except to require that the same $\mathbb{O}$ which rotates to couple $Y_{\mu}$ to hypercharge simultaneously diagonalizes $\mathbb{M}^{2}$ so that

$$
\overline{\mathbb{M}^{2}}=\operatorname{diag}\left(0, M^{\prime 2}, M^{\prime \prime 2}\right)
$$

This implies that the original $\mathbb{M}^{2}$ in the flavor basis is given by

$$
\mathbb{M}^{2}=\mathbb{O} \operatorname{diag}\left(0, M^{\prime 2}, M^{\prime \prime 2}\right) \mathbb{O}^{T},
$$

which results in the following baroque matrix:

$$
\mathbb{M}^{2}=\left(\begin{array}{lll}
a & b & c \\
b & d & e \\
c & e & f
\end{array}\right),
$$


where

$$
\begin{gathered}
a=M^{\prime 2}\left(C_{\psi} S_{\theta} S_{\phi}-C_{\phi} S_{\psi}\right)^{2}+M^{\prime \prime 2}\left(C_{\phi} C_{\psi} S_{\theta}+S_{\phi} S_{\psi}\right)^{2}, \\
b=\left(M^{\prime 2}-M^{\prime \prime 2}\right) C_{\phi} C_{2 \psi} S_{\theta} S_{\phi}+C_{\phi}^{2} C_{\psi}\left(-M^{\prime 2}+M^{\prime \prime 2} S_{\theta}^{2}\right) S_{\psi}+C_{\psi}\left(-M^{\prime \prime 2}+M^{\prime 2} S_{\theta}^{2}\right) S_{\phi}^{2} S_{\psi}, \\
c=C_{\theta}\left[M^{\prime \prime 2} C_{\phi}^{2} C_{\psi} S_{\theta}+M^{\prime 2} C_{\psi} S_{\theta} S_{\phi}^{2}-\left(M^{\prime 2}-M^{\prime \prime 2}\right) C_{\phi} S_{\phi} S_{\psi}\right] \\
d=M^{\prime \prime 2}\left(C_{\psi} S_{\phi}-C_{\phi} S_{\theta} S_{\psi}\right)^{2}+M^{\prime 2}\left(C_{\phi} C_{\psi}+S_{\theta} S_{\phi} S_{\psi}\right)^{2}, \\
e=C_{\theta}\left[\left(M^{\prime 2}-M^{\prime \prime 2}\right) C_{\phi} C_{\psi} S_{\phi}+M^{\prime \prime 2} C_{\phi}^{2} S_{\theta} S_{\psi}+M^{\prime 2} S_{\theta} S_{\phi}^{2} S_{\psi}\right] \\
f=C_{\theta}^{2}\left(M^{\prime \prime 2} C_{\phi}^{2}+M^{\prime 2} S_{\phi}^{2}\right) .
\end{gathered}
$$

We turn now to discuss the phenomenological aspects of anomalous $U(1)$ gauge bosons related to experimental searches for new physics at the Tevatron and at the CERN's Large Hadron Collider (LHC).

\section{Search for New Gauge Bosons at Hadron Colliders}

Taken at face value, the disparity between CDF [18-21] and DØ [22] results insinuates a commodious uncertainty as to whether there is an excess of events in the dijet system invariant mass distribution of the associated production of a $W$ boson with 2 jets (hereafter $W_{j j}$ production). The $M_{j j}$ excess showed up in $4.3 \mathrm{fb}^{-1}$ of integrated luminosity collected with the CDF detector as a broad bump between about 120 and $160 \mathrm{GeV}[18,19]$. The CDF collaboration fitted the excess (hundreds of events in the $\ell j j+E_{T}^{\prime}$ channel) to a Gaussian and estimated its production cross-section times, the dijet branching ratio, to be $4 \mathrm{pb}$. This is roughly 300 times the SM Higgs rate $\sigma(p \bar{p} \rightarrow W H) \times \mathrm{BR}(H \rightarrow b \bar{b})$. For a search window of 120-200 GeV, the excess significance above SM background (including systematics uncertainties) has been reported to be $3.2 \sigma[18,19]$. Recently, CDF has included an additional $3 \mathrm{fb}^{-1}$ to their data sample, for a total of $7.3 \mathrm{fb}^{-1}$, and the statistical significance has grown to $\sim 4.8 \sigma$ ( $\sim 4.1 \sigma$ including systematics) $[20,21]$. More recently, the D $\varnothing$ collaboration released an analysis (which closely follows the CDF analysis) of their $W_{j j}$ data finding "no evidence for anomalous resonant dijet production" [22]. Using an integrated luminosity of $4.3 \mathrm{fb}^{-1}$, they set a 95\% CL upper limit of $1.9 \mathrm{pb}$ on a resonant $W_{j j}$ production cross section.

Although various explanations have been proposed for the CDF anomaly [23-34], perhaps the simplest is the introduction of a new leptophobic $Z^{\prime}$ gauge boson [35-44]. The suppressed coupling to leptons (or more specifically, to electrons and muons) is required to evade the strong constraints of the Tevatron $Z^{\prime}$ searches in the dilepton mode [4547] and LEP-II measurements of $e^{+} e^{-} \rightarrow e^{+} e^{-}$above the Z-pole [48-50]. In complying with the precision demanded of our phenomenological approach, it would be sufficient to consider a $1 \%$ branching fraction to leptons as consistent with the experimental bound. This approximation is within a factor of a few of model independent published experimental bounds. In addition, the mixing of the $Z^{\prime}$ with the SM $Z$ boson should be extremely small to be compatible with precision measurements at the Z-pole by the LEP experiments [51]. 
All existing dijet-mass searches via direct production at the Tevatron are limited to $M_{j j}>200 \mathrm{GeV}$ [52-55] and therefore cannot constrain the existence of a $Z^{\prime}$ with $M_{Z^{\prime}} \simeq$ $150 \mathrm{GeV}$. The strongest constraint on a light leptophobic $Z^{\prime}$ comes from the dijet search by the UA2 collaboration, which has placed a $90 \%$ CL upper bound on $\sigma\left(p \bar{p} \rightarrow Z^{\prime}\right) \times \mathrm{BR}\left(Z^{\prime} \rightarrow j j\right)$ in this energy range $[56,57]$. A comprehensive model independent analysis incorporating Tevatron and UA2 data to constrain the $Z^{\prime}$ parameters for predictive purposes at the LHC was recently presented [58]. (Other phenomenological restrictions on $Z^{\prime}$-gauge bosons were recently discussed in [59].) As of today, the ATLAS and CMS experiments are not sensitive to the $W_{j j}$ signal $[60,61]$. However, LHC will eventually weigh in on this issue: if new physics is responsible for the CDF anomaly, an excess in $\ell j j+Z_{T}$ should become statistically significant in ATLAS and CMS by the end of the year [62].

As usual, the $U(1)$ gauge interactions arise through the covariant derivative

$$
\Phi_{\mu}=\partial_{\mu}-i g_{3}^{\prime} C_{\mu} Q_{3}-i g_{4}^{\prime} \widetilde{B}_{\mu} Q_{1 L}-i g_{1}^{\prime} B_{\mu} Q_{1 R}
$$

where $g_{1}^{\prime}, g_{3}^{\prime}$, and $g_{4}^{\prime}$ are the gauge coupling constants. The fields $C_{\mu}, \widetilde{B}_{\mu}, B_{\mu}$ are related to $Y_{\mu}, Y_{\mu}^{\prime}$, and $Y_{\mu}^{\prime \prime}$ by the rotation matrix,

$$
\mathbb{O}=\left(\begin{array}{ccc}
C_{\theta} C_{\psi} & -C_{\phi} S_{\psi}+S_{\phi} S_{\theta} C_{\psi} & S_{\phi} S_{\psi}+C_{\phi} S_{\theta} C_{\psi} \\
C_{\theta} S_{\psi} & C_{\phi} C_{\psi}+S_{\phi} S_{\theta} S_{\psi} & -S_{\phi} C_{\psi}+C_{\phi} S_{\theta} S_{\psi} \\
-S_{\theta} & S_{\phi} C_{\theta} & C_{\phi} C_{\theta}
\end{array}\right),
$$

with Euler angles $\theta, \psi$, and $\phi$. Equation (4.1) can be rewritten in terms of $Y_{\mu}, Y_{\mu}^{\prime}$, and $Y_{\mu}^{\prime \prime}$ as follows:

$$
\begin{aligned}
\Phi_{\mu}= & \partial_{\mu}-i Y_{\mu}\left(-S_{\theta} g_{1}^{\prime} Q_{1 R}+C_{\theta} S_{\psi} g_{4}^{\prime} Q_{1 L}+C_{\theta} C_{\psi} g_{3}^{\prime} Q_{3}\right) \\
& -i Y_{\mu}^{\prime}\left[C_{\theta} S_{\phi} g_{1}^{\prime} Q_{1 R}+\left(C_{\phi} C_{\psi}+S_{\theta} S_{\phi} S_{\psi}\right) g_{4}^{\prime} Q_{1 L}+\left(C_{\psi} S_{\theta} S_{\phi}-C_{\phi} S_{\psi}\right) g_{3}^{\prime} Q_{3}\right] \\
& -i Y_{\mu}^{\prime \prime}\left[C_{\theta} C_{\phi} g_{1}^{\prime} Q_{1 R}+\left(-C_{\psi} S_{\phi}+C_{\phi} S_{\theta} S_{\psi}\right) g_{4}^{\prime} Q_{1 L}+\left(C_{\phi} C_{\psi} S_{\theta}+S_{\phi} S_{\psi}\right) g_{3}^{\prime} Q_{3}\right] .
\end{aligned}
$$

Now, by demanding that $Y_{\mu}$ has the hypercharge $Q_{Y}$ given in (1.4) we fix the first column of the rotation matrix $\mathbb{O}$ :

$$
\left(\begin{array}{c}
C_{\mu} \\
\widetilde{B}_{\mu} \\
B_{\mu}
\end{array}\right)=\left(\begin{array}{c}
Y_{\mu} \frac{c_{3} g_{\Upsilon}}{g_{3}^{\prime}} \cdots \\
Y_{\mu} \frac{c_{4} g_{\Upsilon}}{g_{4}^{\prime}} \cdots \\
Y_{\mu} \frac{c_{1} g_{Y}}{g_{1}^{\prime}} \cdots
\end{array}\right),
$$


and we determine the value of the two associated Euler angles

$$
\begin{gathered}
\theta=-\arcsin \left[\frac{c_{1} g_{Y}}{g_{1}^{\prime}}\right], \\
\psi=\arcsin \left[\frac{c_{4} g_{Y}}{\left(g_{4}^{\prime} C_{\theta}\right)}\right] .
\end{gathered}
$$

The couplings $g_{1}^{\prime}$ and $g_{4}^{\prime}$ are related through the orthogonality condition (2.11),

$$
\left(\frac{c_{4}}{g_{4}^{\prime}}\right)^{2}=\frac{1}{g_{Y}^{2}}-\left(\frac{c_{3}}{g_{3}^{\prime}}\right)^{2}-\left(\frac{c_{1}}{g_{1}^{\prime}}\right)^{2}
$$

with $g_{3}^{\prime}$ fixed by the relation for $U(N)$ unification: $g_{3}^{\prime}=\sqrt{6} g_{3}$. In what follows, we take $5 \mathrm{TeV}$ as a reference point for running down to $150 \mathrm{GeV}$ the $g_{3}^{\prime}$ coupling using (2.13), that is, ignoring mass threshold effects. This yields $g_{3}^{\prime}=0.383$. We have checked that the running of the $g_{3}^{\prime}$ coupling does not change significantly by varying the scale of $U(N)$ unification between $3 \mathrm{TeV}$ and $10 \mathrm{TeV}$.

The phenomenological analysis thus far has been formulated in terms of the massdiagonal basis set of gauge fields $\left(Y, Y^{\prime}, Y^{\prime \prime}\right)$. As a result of the electroweak phase transition, the coupling of this set with the Higgses will induce mixing, resulting in a new mass-diagonal basis set $\left(Z, Z^{\prime}, Z^{\prime \prime}\right)$. It will suffice to analyze only the $2 \times 2$ system $\left(Y, Y^{\prime}\right)$ to see that the effects of this mixing are totally negligible. We consider simplified zeroth and first order (mass) ${ }^{2}$ matrices

$$
\left(M^{2}\right)^{(0)}=\left(\begin{array}{cc}
0 & 0 \\
0 & M^{\prime 2}
\end{array}\right)\left(M^{2}\right)^{(1)}=\left(\begin{array}{cc}
\bar{M}_{Z}^{2} & \epsilon \\
\epsilon & m^{\prime 2}
\end{array}\right),
$$

where $M^{\prime}$ is the mass of the $Y^{\prime}$ gauge field, $\bar{M}_{Z}=\sqrt{g_{2}^{2}+g_{Y}^{2}} v / 2$ is the usual tree level formula for the mass of the $Z$ particle in the electroweak theory (before mixing), $g_{2} \simeq 0.651$ is the electroweak coupling constant, $v$ is the vacuum expectation value of the Higgs field, $g_{Y} \simeq$ 0.357 , and $\epsilon, m^{\prime 2}$ are of $\mathcal{O}\left(\bar{M}_{Z}^{2}\right)$.

Standard Rayleigh-Schrodinger perturbation theory then provides the (mass) ${ }^{2}$ (to second order in $\bar{M}_{Z}^{2}$ ) and wave functions (to first order) of the mass-diagonal eigenfields $\left(Z, Z^{\prime}\right)$ corresponding to $\left(Y, Y^{\prime}\right)$,

$$
\begin{gathered}
M_{Z}^{2}=\bar{M}_{Z}^{2}-\left(\frac{\epsilon^{2}}{M^{\prime 2}}\right), \quad M_{Z^{\prime}}^{2}=M^{\prime 2}+m^{\prime 2}+\left(\frac{\epsilon^{2}}{M^{\prime 2}}\right), \\
Z=Y-\left(\frac{\epsilon}{M^{\prime 2}}\right) Y^{\prime}, \quad Z^{\prime}=Y^{\prime}+\left(\frac{\epsilon}{M^{\prime 2}}\right) Y .
\end{gathered}
$$


From (4.8) the shift in the mass of the $Z$ is given by $\delta M_{Z}^{2}=\left(\epsilon / M^{\prime}\right)^{2}$, so that $\epsilon=$ $M^{\prime} \sqrt{2 M_{Z} \delta M_{Z}}$. The admixture of $Y$ in the mass-diagonal field $Z^{\prime}$ is

$$
\theta=\frac{\epsilon}{M^{\prime 2}}=\frac{M_{Z}}{M^{\prime}} \sqrt{\frac{2 \delta M_{Z}}{M_{Z}}} \simeq 0.004
$$

Since all effects go as $\theta^{2} \simeq 1.6 \times 10^{-5}$, all further discussion will be, with negligible error, in terms of $Z^{\prime}$. By the same token, the admixture of $Y^{\prime}$ in the eigenfield $Z$ is negligible, so that the discussion henceforth will reflect $Z \simeq Y$ and $\bar{M}_{Z}^{2} \simeq M_{Z}^{2}$.

The $f \bar{f} Z^{\prime}$ Lagrangian is of the form

$$
\begin{aligned}
\mathcal{L} & =\frac{1}{2} \sqrt{g_{Y}^{2}+g_{2}^{2}} \sum_{f}\left(\epsilon_{f_{L}} \bar{\psi}_{f_{L}} \gamma^{\mu} \psi_{f_{L}}+\epsilon_{f_{R}} \bar{\psi}_{f_{R}} \gamma^{\mu} \psi_{f_{R}}\right) Z_{\mu}^{\prime} \\
& =\sum_{f}\left(\left(g_{Y^{\prime}} Q_{Y^{\prime}}\right)_{f_{L}} \bar{\psi}_{f_{L}} \gamma^{\mu} \psi_{f_{L}}+\left(g_{\gamma^{\prime}} Q_{Y^{\prime}}\right)_{f_{R}} \bar{\psi}_{f_{R}} \gamma^{\mu} \psi_{f_{R}}\right) Z_{\mu^{\prime}}^{\prime}
\end{aligned}
$$

where each $\psi_{f_{L(R)}}$ is a fermion field with the corresponding $\gamma^{\mu}$ matrices of the Dirac algebra, and $\epsilon_{f_{L}, f_{R}}=v_{q} \pm a_{q}$, with $v_{q}$ and $a_{q}$, the vector and axial couplings, respectively. The (precut) $W_{j j}$ production rate at the Tevatron $\sqrt{s}=1.96 \mathrm{pb}$, for arbitrary couplings and $M_{Z^{\prime}} \simeq 150 \mathrm{GeV}$, is found to be [58]

$$
\sigma\left(p \bar{p} \longrightarrow W Z^{\prime}\right) \times \mathrm{BR}\left(Z^{\prime} \longrightarrow j j\right) \simeq\left[0.719\left(\epsilon_{u_{L}}^{2}+\epsilon_{d_{L}}^{2}\right)+5.083 \epsilon_{u_{L}} \epsilon_{d_{L}}\right] \times \Gamma\left(\phi, g_{1}^{\prime}\right)_{Z^{\prime} \rightarrow q \bar{q}} p b
$$

where $\Gamma\left(\phi, g_{1}^{\prime}\right)_{Z^{\prime} \rightarrow q \bar{q}}$ is the hadronic branching fraction. The presence of a $W$ in the process shown in Figure 1 restricts the contribution of the quarks to be purely left-handed. Since $\epsilon_{u_{L}}=\epsilon_{d_{L}}$ and the required branching to quarks is above about $99 \%$ (after selection cuts are accounted for), the coupling strength $\epsilon_{q_{L}}^{2}$ is fixed by the $W_{j j}$ production rate. Below, we avoid reference to specific experimental selection cuts and present results for a generous range of possibilities consistent with existing data. [58]:

The dijet production rate at the UA2 $\sqrt{s}=630 \mathrm{GeV}$ can be parametrized as follows

$$
\sigma\left(p \bar{p} \longrightarrow Z^{\prime}\right) \times \mathrm{BR}\left(Z^{\prime} \longrightarrow j j\right) \simeq \frac{1}{2}\left[773\left(\epsilon_{u_{L}}^{2}+\epsilon_{u_{R}}^{2}\right)+138\left(\epsilon_{d_{L}}^{2}+\epsilon_{d_{R}}^{2}\right)\right] \times \Gamma\left(\phi, g_{1}^{\prime}\right)_{Z^{\prime} \rightarrow q \bar{q}} \mathrm{pb}
$$

(our numerical calculation [5] using CTEQ6 [63] agrees within 5\% with the result of [58]). The maximum allowed value of the $\epsilon_{u_{R}}$ and $\epsilon_{d_{R}}$ couplings consistent with the UA2 upper limit is shown in Figure 2. The dilepton production rate at UA2 energies is given by

$$
\sigma\left(p \bar{p} \longrightarrow Z^{\prime}\right) \times \mathrm{BR}\left(Z^{\prime} \longrightarrow \ell \bar{\ell}\right) \simeq \frac{1}{2}\left[773\left(\epsilon_{u_{L}}^{2}+\epsilon_{u_{R}}^{2}\right)+138\left(\epsilon_{d_{L}}^{2}+\epsilon_{d_{R}}^{2}\right)\right] \times \Gamma\left(\phi, g_{1}^{\prime}\right)_{Z^{\prime} \rightarrow \ell \bar{\ell}} \mathrm{pb}
$$




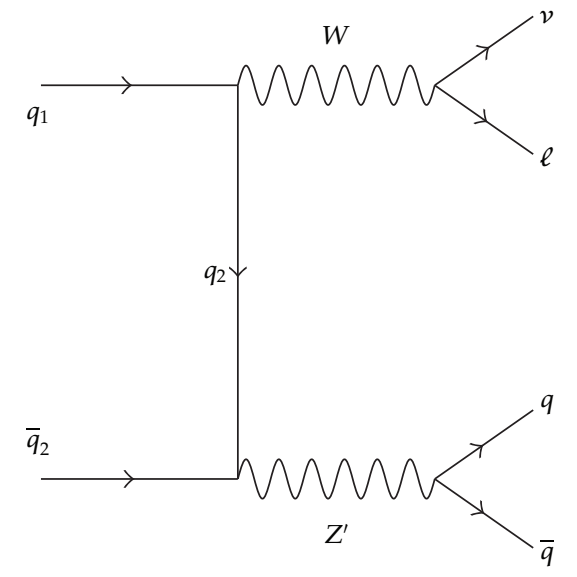

Figure 1: Feynman diagram for $q \bar{q} \rightarrow W Z^{\prime} \rightarrow v \ell j j$.

where $\Gamma\left(\phi, g_{1}^{\prime}\right)_{Z^{\prime} \rightarrow \ell \bar{\ell}}$ is the leptonic branching fraction. From (4.3) and (4.10), we obtain the explicit form of the chiral couplings in terms of $\phi$ and $g_{1}^{\prime}$ :

$$
\begin{gathered}
\epsilon_{u_{L}}=\epsilon_{d_{L}}=\frac{2}{\sqrt{g_{Y}^{2}+g_{2}^{2}}}\left(C_{\psi} S_{\theta} S_{\psi}-C_{\phi} S_{\psi}\right) g_{3}^{\prime} \\
\epsilon_{u_{R}}=-\frac{2}{\sqrt{g_{Y}^{2}+g_{2}^{2}}}\left[C_{\theta} S_{\phi} g_{1}^{\prime}+\left(C_{\psi} S_{\theta} S_{\psi}-C_{\phi} S_{\psi}\right) g_{3}^{\prime}\right], \\
\epsilon_{d_{R}}=\frac{2}{\sqrt{g_{Y}^{2}+g_{2}^{2}}}\left[C_{\theta} S_{\phi} g_{1}^{\prime}-\left(C_{\psi} S_{\theta} S_{\psi}-C_{\phi} S_{\psi}\right) g_{3}^{\prime}\right] .
\end{gathered}
$$

The second strong constraint on the model derives from the mixing of the $Z$ and the $Y^{\prime}$ through their coupling to the Higgs doublet. The last two terms in the covariant derivative

$$
\boldsymbol{\Phi}_{\mu}=\partial_{\mu}-i \frac{1}{\sqrt{g_{2}^{2}+g_{Y}^{2}}} Z_{\mu}\left(g_{2}^{2} T^{3}-g_{Y}^{2} Q_{Y}\right)-i g_{Y^{\prime}} Y_{\mu}^{\prime} Q_{Y^{\prime}}-i g_{Y^{\prime \prime}} Y_{\mu}^{\prime \prime} Q_{Y^{\prime \prime}}
$$

are conveniently written as

$$
-i \frac{x_{H}}{v} \bar{M}_{Z} Y_{\mu}^{\prime}-i \frac{y_{H}}{v} \bar{M}_{Z} Y_{\mu \prime}^{\prime \prime}
$$

where

$$
\begin{aligned}
& x_{H}=1.9 \sqrt{g_{1}^{\prime 2}-0.032} S_{\phi}, \\
& y_{H}=1.9 \sqrt{g_{1}^{\prime 2}-0.032} C_{\phi},
\end{aligned}
$$




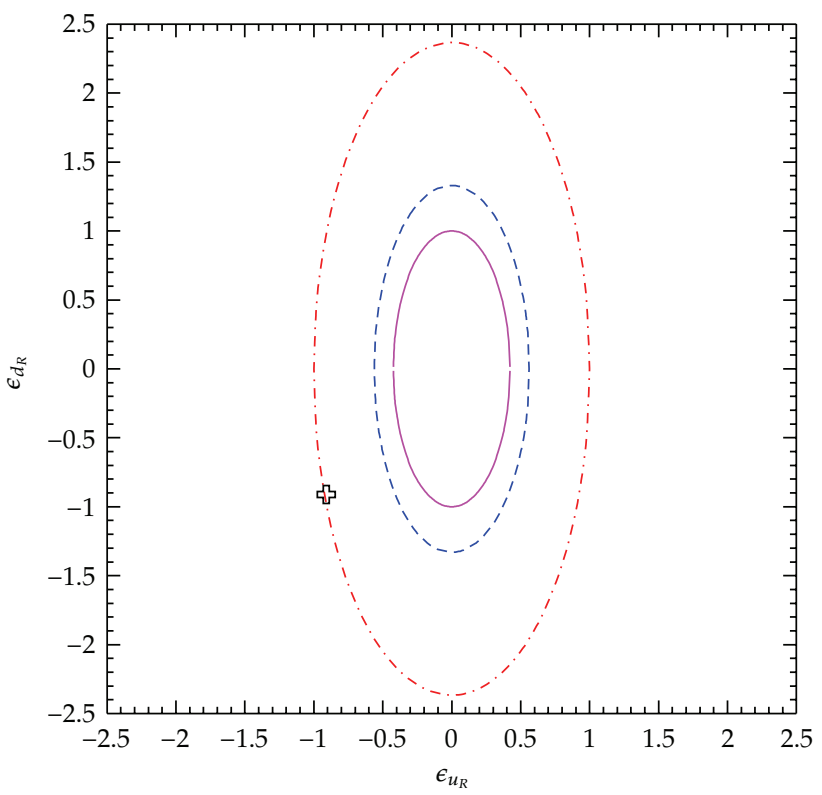

Figure 2: The ellipses show the values of $\epsilon_{u_{R}}$ and $\epsilon_{d_{R}}$ that saturate the UA2 and DØ limits on direct $Z^{\prime} \rightarrow j j$ and $W_{j j}$ production, respectively. The solid ellipse is based on the assumption that experimental selection cuts will cause negligible reduction in event rates, the dashed ellipse corresponds to a reduction in event rates by $50 \%$, and the dot-dashed ellipse corresponds to a $66 \%$ reduction in DØ event rates and $70 \%$ reduction in UA2 event rates. The cross indicates the best eyeball fit that simultaneously ensures small $Z-Z^{\prime}$ mixing and $\Gamma_{Z^{\prime} \rightarrow e^{+} e^{-}} / \Gamma_{Z^{\prime} \rightarrow q \bar{q}} \lesssim 1 \%$.

and $T^{3}=\sigma^{3} / 2$. The Higgs field kinetic term $\left(D_{\mu} H\right)^{\dagger}\left(D_{\mu} H\right)$ together with the anomalous mass terms $\left(-(1 / 2) M^{\prime 2} Y_{\mu}^{\prime} Y^{\prime \mu}-(1 / 2) M^{\prime \prime 2} Y_{\mu}^{\prime \prime} Y^{\prime \prime \mu}\right)$ yields the following mass square matrix (we note in passing that two "supersymmetric" Higgses $H_{u} \equiv H$ and $H_{d}=H^{\dagger}$, with charges $Q_{3}=$ $Q_{1 L}=0, Q_{1 R}=1, Q_{Y}=1 / 2$ and $Q_{3}=Q_{1 L}=0, Q_{1 R}=-1, Q_{Y}=-1 / 2$, would also be sufficient to give masses to all the chiral fermions. Here, $\left\langle H_{u}\right\rangle=\left(\begin{array}{l}0 \\ v_{u}\end{array}\right),\left\langle H_{d}\right\rangle=\left(\begin{array}{c}v_{d} \\ 0\end{array}\right), v=\sqrt{v_{u}^{2}+v_{d}^{2}}$, and $\tan \beta \equiv v_{u} / v_{d}$. It is easily seen that the corresponding mass square matrix is independent of $\tan \beta[5])$ :

$$
\left(\begin{array}{ccc}
\bar{M}_{Z}^{2} & \bar{M}_{Z}^{2} x_{H} & \bar{M}_{Z}^{2} y_{H} \\
\bar{M}_{Z}^{2} x_{H} & \bar{M}_{Z}^{2} x_{H}^{2}+M^{\prime 2} & \bar{M}_{Z}^{2} x_{H} y_{H} \\
\bar{M}_{Z}^{2} y_{H} & \bar{M}_{Z}^{2} x_{H} y_{H} & \bar{M}_{Z}^{2} y_{H}^{2}+M^{\prime \prime 2}
\end{array}\right)
$$

Next, taking $M_{Z^{\prime}}=150 \mathrm{GeV}$ we use the two degrees of freedom of the model $\left(g_{1}^{\prime}, \phi\right)$ to demand the shift of the $Z$ mass to lie within 1 standard deviation of the experimental value and leptophobia. This occurs for $g_{1}^{\prime}=0.2, \phi=0.0028$, and $M_{Z^{\prime \prime}}=5 \mathrm{TeV}$, corresponding to a suppression $\Gamma_{Z^{\prime \prime} \rightarrow e^{+} e^{-}} / \Gamma_{Z^{\prime \prime} \rightarrow q \bar{q}} \simeq 1 \%$ [5]. This also corresponds to $\theta=-1.103, \psi=-1.227$, and $g_{4}^{\prime}=0.42$. The $g_{Y^{\prime}} Q_{Y^{\prime}}$ and $g_{Y^{\prime \prime}} Q_{Y^{\prime \prime}}$ couplings to the chiral fields are fixed and given in Table 2. 
Table 2: Chiral couplings of $Y^{\prime}$ and $Y^{\prime \prime}$ gauge bosons for $\phi=0.0028$ and $g_{1}^{\prime}=0.2$.

\begin{tabular}{lcc}
\hline Name & $g_{Y^{\prime}} Q_{Y^{\prime}}$ & $g_{\gamma^{\prime \prime}} Q_{\Upsilon^{\prime \prime}}$ \\
\hline$Q_{i}$ & 0.368 & -0.119 \\
$U_{i}$ & 0.368 & -0.028 \\
$D_{i}$ & 0.368 & -0.209 \\
$L_{i}$ & 0.143 & 0.143 \\
$E_{i}$ & 0.142 & 0.262 \\
$N_{i}$ & 0.143 & 0.443 \\
\hline
\end{tabular}

The accompanying values of $\epsilon_{u_{R}}$ and $\epsilon_{d_{R}}$ are shown in Figure 2. Now, substituting the above figures into (4.4), we obtain the projections over $Y, Y^{\prime}, Y^{\prime \prime}$ :

$$
\begin{aligned}
& Y=1.8 \times 10^{-1} Q_{1 R}+5.9 \times 10^{-2} Q_{3}-1.8 \times 10^{-1} Q_{1 L} \\
& Y^{\prime}=2.5 \times 10^{-4} Q_{1 R}+3.7 \times 10^{-1} Q_{3}+1.4 \times 10^{-1} Q_{1 L} \\
& Y^{\prime \prime}=9.0 \times 10^{-2} Q_{1 R}-1.2 \times 10^{-1} Q_{3}+3.5 \times 10^{-1} Q_{1 L}
\end{aligned}
$$

Using (1.6) it is straightforward to see that $Z^{\prime}$ and $Z^{\prime \prime}$ become essentially $B$ and $B-L$, respectively.

The $Z^{\prime}$ couplings to quarks lead to a large (precut) $W_{j j}$ production $(\simeq 6 \mathrm{pb})$ at the Tevatron and at $\sqrt{s}=630 \mathrm{GeV}$, a direct (precut) $Z^{\prime} \rightarrow j j$ production $(\simeq 700 \mathrm{pb})$ in the region excluded by UA2 data. However, it is worthwhile to point out that the UA2 collaboration performed their analysis in the early days of QCD jet studies. Their upper bound depends crucially on the quality of the Monte Carlo and detector simulation which are primitive by today's standard. They also use events with two exclusive jets, where jets were constructed using an infrared unsafe jet algorithm [64]. In view of the considerable uncertainties associated with the UA2 analysis, we remain skeptical of drawing negative conclusions. Instead we argue that our model [5] could provide an explanation of the CDF anomaly if acceptance and pseudorapidity cuts reduce the $W_{j j}$ production rate by about $35-66 \%$ and the UA2 90\% CL bound is taken as an order-of-magnitude limit (Similar arguments have been previously advocated by Nelson et al. in [23-34] and by Liu et al. in [35-44]).

Since the CDF signal is in dispute, it is of interest to study the predictions of the model for a leptophobic $Z^{\prime}$ at energies not obtainable at the Tevatron, but within the range of the LHC. The ATLAS collaboration has searched for narrow resonances in the invariant mass spectrum of dimuon and dielectron final states in event samples corresponding to an integrated luminosity of $1.21 \mathrm{fb}^{-1}$ and $1.08 \mathrm{fb}^{-1}$, respectively [66]. The spectra are consistent with SM expectations and thus a lower mass limit of $1.83 \mathrm{TeV}$ on the sequential SM $Z^{\prime}$ has been set. (In the sequential SM the $Z^{\prime}$ has the same couplings to fermions as the $Z$ boson.) Therefore, for $M_{Z^{\prime}} \geq 1 \mathrm{TeV}$, we scan the $g_{1}^{\prime}-\phi$ parameter space demanding the shift of the $Z$ mass to lie within 1 standard deviation of the experimental value and small $(\lesssim 1 \%)$ branching to leptons. We find that for $g_{1}^{\prime}=0.195, \phi=-0.0638$, and $M_{Z^{\prime \prime}} \geq 2 M_{Z^{\prime}}$, the ratio $\Gamma_{Z^{\prime} \rightarrow e^{+} e^{-}} / \Gamma_{Z^{\prime} \rightarrow q \bar{q}} \lesssim 1 \%$ [5]. The chiral couplings to the $Z^{\prime}$ and $Z^{\prime \prime}$ gauge bosons for these fiducial values are given in Table 3. Again, we see that $Z^{\prime}$ and $Z^{\prime \prime}$ are essentially $B$ and $B-L$. 
Table 3: Chiral couplings of $Y^{\prime}$ and $Y^{\prime \prime}$ gauge bosons for $\phi=-0.0638$ and $g_{1}^{\prime}=0.195$.

\begin{tabular}{lcc}
\hline Name & $g_{\gamma^{\prime}} Q_{\gamma^{\prime}}$ & $g_{\gamma^{\prime \prime}} Q_{\gamma^{\prime \prime}}$ \\
\hline$Q_{i}$ & 0.370 & -0.112 \\
$U_{i}$ & 0.365 & -0.033 \\
$D_{i}$ & 0.375 & -0.190 \\
$L_{i}$ & 0.154 & 0.154 \\
$E_{i}$ & 0.159 & 0.338 \\
$N_{i}$ & 0.149 & 0.495 \\
\hline
\end{tabular}

The decay width of $Z^{\prime} \rightarrow f \bar{f}$ is given by [67]

$$
\Gamma\left(Z^{\prime} \longrightarrow f \bar{f}\right)=\frac{G_{F} M_{Z}^{2}}{6 \pi \sqrt{2}} N_{C} C\left(M_{Z^{\prime}}^{2}\right) M_{Z^{\prime}} \sqrt{1-4 x}\left[v_{f}^{2}(1+2 x)+a_{f}^{2}(1-4 x)\right]
$$

where $G_{F}$ is the Fermi coupling constant, $C\left(M_{Z^{\prime}}^{2}\right)=1+\alpha_{S} / \pi+1.409\left(\alpha_{s} / \pi\right)^{2}-12.77\left(\alpha_{s} / \pi\right)^{3}$, $\alpha_{s}=\alpha_{S}\left(M_{Z^{\prime}}\right)$ is the strong coupling constant at the scale $M_{Z^{\prime}}, x=m_{f}^{2} / M_{Z^{\prime}}^{2}, v_{f}$ and $a_{f}$ are the vector and axial couplings, and $N_{C}=3$ or 1 if $f$ is a quark or a lepton, respectively. Using the fiducial values of $g_{1}^{\prime}$ and $\phi$ fitted in Table 2 , for $M_{Z^{\prime}}=1 \mathrm{TeV}$, we obtain $\Gamma=60.9 \mathrm{TeV}$. Hence, to compare our predictions (at the parton level) with LHC experimental searches in dilepton and dijets, it is sufficient to consider the production cross section in the narrow $Z^{\prime}$ width approximation,

$$
\widehat{\sigma}\left(q \bar{q} \longrightarrow Z^{\prime}\right)=K \frac{2 \pi}{3} \frac{G_{F} M_{Z}^{2}}{\sqrt{2}}\left[v_{q}^{2}\left(\phi, g_{1}^{\prime}\right)+a_{q}^{2}\left(\phi, g_{1}^{\prime}\right)\right] \delta\left(\widehat{s}-M_{Z^{\prime}}^{2}\right)
$$

where the $K$-factor represents the enhancement from higher order QCD processes estimated to be $K \simeq 1.3$ [68]. After folding $\widehat{\sigma}$ with the CTEQ6 parton distribution functions [63], we determine (at the parton level) the resonant production cross section. In Figure 3 we compare the predicted $\sigma\left(p \bar{p} \rightarrow Z^{\prime}\right) \times \mathrm{BR}\left(Z^{\prime} \rightarrow \ell \ell\right)\left(\right.$ a) and $\sigma\left(p \bar{p} \rightarrow Z^{\prime}\right) \times \mathrm{BR}\left(Z^{\prime} \rightarrow j j\right)$ (b) production rates with 95\% CL upper limits recently reported by the ATLAS [66] and CMS [65] collaborations. Selection cuts will probably reduce event rates by factors of 2 to 3 . Keeping this in mind, we conclude that the $2012 \mathrm{LHC7}$ run will probe $3 \mathrm{TeV}<M_{Z^{\prime}}<4 \mathrm{TeV}$, whereas future runs from LHC14 will provide a generous discovery potential of up to about $M_{Z^{\prime}} \sim 8 \mathrm{TeV}$.

We turn now to discuss the string origin and the compelling properties of the $U(3)_{C} \times$ $S p(1)_{L} \times U(1)_{L} \times U(1)_{R}$ gauge group.

\section{Perturbative D-Brane Models in a Nutshell}

At the time of its formulation and for years thereafter, Superstring Theory was regarded as a unifying framework for Planck-scale quantum gravity and TeV-scale SM physics. Important advances were fueled by the realization of the vital role played by D-branes $[69,70]$ in connecting string theory to phenomenology. This has permitted the formulation [71] of string theories with compositeness setting in $\mathrm{TeV}$ scales and large extra dimensions, see 


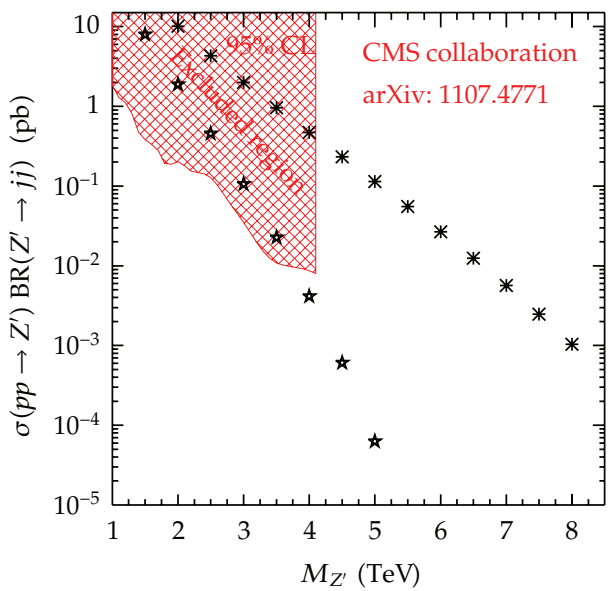

(Precut) $Z^{\prime}$ signal

$\star$ LHC7

* LHC14

(a)

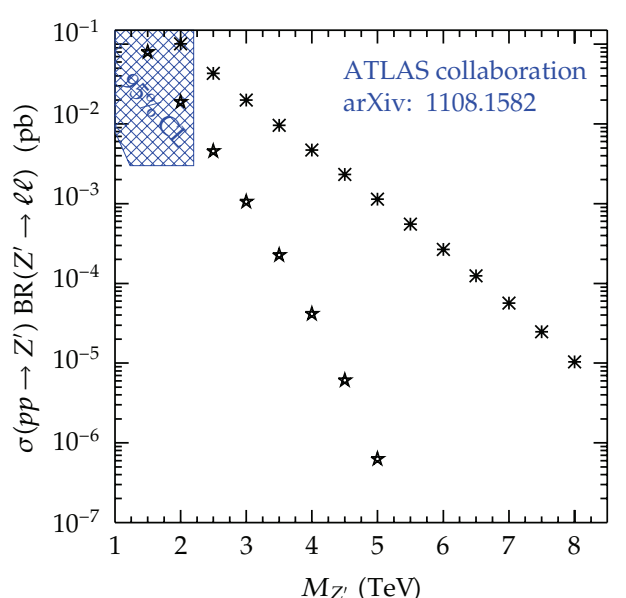

(Precut) $Z^{\prime}$ signal

$\star$ LHC7

* LHC14

(b)

Figure 3: Comparison of the (precut) total cross section for the production of $p p \rightarrow Z^{\prime} \rightarrow j j$ (a) and $p p \rightarrow Z^{\prime} \rightarrow \ell \ell(b)$ with the $95 \%$ CL upper limits on the production of a gauge boson decaying into two jets (a) and two leptons (b), as reported by the CMS (corrected by acceptance) [65] and ATLAS [66] collaborations, respectively. We have taken $\phi=-0.0638, g_{1}^{\prime}=0.195$. For isotropic decays (independently of the resonance), the acceptance for the CMS detector has been reported to be $\mathcal{A} \approx 0.6$ [65]. The predicted $Z^{\prime}$ production rates are shown for $\sqrt{s}=7 \mathrm{TeV}$ and $\sqrt{s}=14 \mathrm{TeV}$.

Appendix A. There are two paramount phenomenological consequences for TeV scale Dbrane string physics: the emergence of Regge recurrences at parton collision energies $\sqrt{\widehat{s}} \sim$ string scale $\equiv M_{s}$ and the presence of one or more additional $U(1)$ gauge symmetries, beyond the $U(1)_{Y}$ of the SM.

D-brane TeV-scale string compactifications provide a collection of building block rules that can be used to build up the SM or something very close to it [72-82]. The details of the D-brane construct depend a lot on whether we use oriented string or unoriented string models. The basic unit of gauge invariance for oriented string models is a $U(1)$ field, so that a stack of $N$ identical D-branes eventually generates a $U(\mathrm{~N})$ theory with the associated $U(N)$ gauge group. In the presence of many D-brane types, the gauge group becomes a product form $\Pi U\left(N_{i}\right)$, where $N_{i}$ reflects the number of D-branes in each stack. Gauge bosons (and associated gauginos in a SUSY model) arise from strings terminating on one stack of Dbranes, whereas chiral matter fields are obtained from strings stretching between two stacks. Each of the two strings end points carries a fundamental charge with respect to the stack of branes on which it terminates. Matter fields thus possess quantum numbers associated with a bifundamental representation. In orientifold brane configurations, which are necessary for tadpole cancellation, and thus consistency of the theory, open strings become in general nonoriented. For unoriented strings, the above rules still apply, but we are allowed many more choices because the branes come in two different types. There are the branes whose images under the orientifold are different from themselves and their image branes, and also branes which are their own images under the orientifold procedure. Stacks of the first type 
combine with their mirrors and give rise to $U(N)$ gauge groups, while stacks of the second type give rise to only $S O(N)$ or $S p(N)$ gauge groups.

The minimal embedding of the SM particle spectrum requires at least three brane stacks [12] leading to three distinct models of the type $U(3)_{C} \times U(2)_{L} \times U(1)$ that were classified in $[12,83]$. Only one of them (model $C$ of $[83]$ ) has baryon number as symmetry that guarantees proton stability (in perturbation theory) and can be used in the framework of TeV strings. Moreover, since $Q_{2}$ (associated to the $U(1)$ of $\left.U(2)_{L}\right)$ does not participate in the hypercharge combination, $U(2)_{L}$ can be replaced by $S p(1)_{L}$ leading to a model with one extra $U(1)$, the baryon number, besides hypercharge $[84,85]$. Since baryon number is anomalous, the extra abelian gauge field becomes massive by the Green-Schwarz mechanism [86-90], behaving at low energies as a $Z^{\prime}$ with a mass in general lower than the string scale by an order of magnitude corresponding to a loop factor [91, 92]. Lepton number is not a symmetry creating a problem with large neutrino masses through the Weinberg dimensionfive operator $L L H H$ suppressed only by the TeV string scale.

The SM embedding in four D-brane stacks leads to many more models that have been classified in $[13,93]$. In order to make a phenomenologically interesting choice, we focus on models where $U(2)_{L}$ can be reduced to $S p(1)$. The minimal SM extension build up out of four stacks of D-branes is $U(3)_{C} \times S p(1)_{L} \times U(1)_{L} \times U(1)_{R}$. A schematic representation of the D-brane structure is shown in Figure 4. The corresponding fermion quantum numbers are given in Table 1 . Recall that the combination $B-L$ is anomaly free. As mentioned already, anomalous $U(1)$ 's become massive necessarily due to the Green-Schwarz anomaly cancellation, but nonanomalous $U(1)$ 's can also acquire masses due to effective sixdimensional anomalies associated for instance to sectors preserving $N=2$ SUSY [91, 92]. (In fact, also the hypercharge gauge boson of $U(1)_{Y}$ can acquire a mass through this mechanism. In order to keep it massless, certain topological constraints on the compact space have to be met.) These two-dimensional "bulk" masses become therefore larger than the localized masses associated to four-dimensional anomalies, in the large volume limit of the two extra dimensions. Specifically for D $p$-branes with $(p-3)$-longitudinal compact dimensions the masses of the anomalous and, respectively, the nonanomalous $U(1)$ gauge bosons have the following generic scale behavior:

$$
\begin{aligned}
& \text { anomalous } U(1)_{i}: M_{Z^{\prime}}=g_{i}^{\prime} M_{s} \\
& \text { nonanomalous } U(1)_{i}: M_{Z^{\prime \prime}}=g_{i}^{\prime} M_{s}^{3} V_{2} .
\end{aligned}
$$

Here $g_{i}^{\prime}$ is the gauge coupling constant associated to the group $U(1)_{i}$, given by $g_{i}^{\prime} \propto g_{s} / \sqrt{V_{\|}}$, where $g_{s}$ is the string coupling and $V_{\|}$is the internal D-brane world-volume along the $(p-3)$ compact extra dimensions, up to an order one proportionality constant. Moreover, $V_{2}$ is the internal two-dimensional volume associated to the effective six-dimensional anomalies giving mass to the nonanomalous $U(1)_{i}$. For example, for the case of D5-branes, whose common intersection locus is just 4-dimensional Minkowski-space, $V_{\|}=V_{2}$ denotes the volume of the longitudinal, two-dimensional space along the two internal D5-brane directions. Since internal volumes are bigger than one in string units to have effective field theory description, the masses of nonanomalous $U(1)$-gauge bosons are generically larger than the masses of the anomalous gauge bosons. Since we want to identify the light $Z^{\prime}$ gauge boson with baryon number, which is always anomalous, a hierarchy compared to the second $U(1)$-gauge boson $Z^{\prime \prime}$ can arise, if we identify $Z^{\prime \prime}$ with the anomaly free combination $B-L$, and take the internal world-volume $V_{2}$ a bit larger than the string scale. (In [94] a 


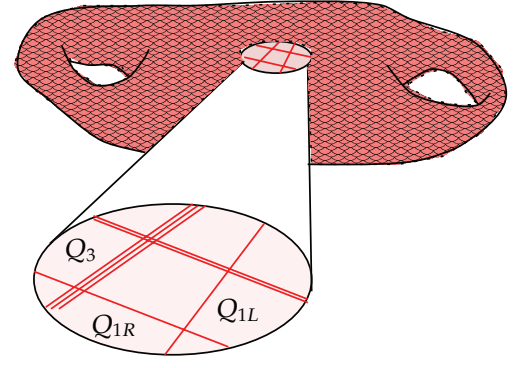

(a)

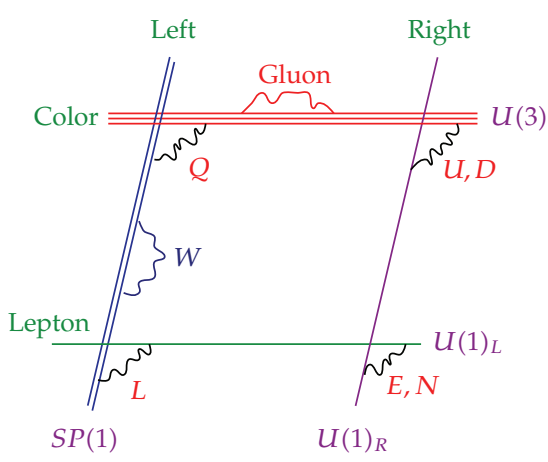

(b)

Figure 4: Pictorial representation of the $U(1)_{C} \times S p(1)_{L} \times U(1)_{L} \times U(1)_{R}$ D-brane model.

different (possibly T-dual) scenario with D7-branes was investigated. In this case the masses of the anomalous and nonanomalous $U(1)$ 's appear to exhibit a dependence on the entire sixdimensional volume, such that the nonanomalous masses become lighter than the anomalous ones.) In principle, this hierarchy can be advocated to explain the $Z^{\prime}-Z^{\prime \prime}$ mass ratio required to explain the CDF anomaly. (It is important to stress that in SUSY models derived from D-brane compactifications there can be a light $Z^{\prime}$ even if the string scale is $\mathcal{O}\left(M_{\mathrm{Pl}}\right)[95,96]$.)

Particles created by vibrations of relativistic strings populate Regge trajectories relating their spins $J$ and masses $M$,

$$
J=J_{0}+\alpha^{\prime} M^{2}
$$

where $\alpha^{\prime}=M_{s}^{-2}$ is the Regge slope parameter. Thus, if $M_{s}$ is of order few TeVs, a whole tower of infinite string excitations will open up at this low mass threshold. Should nature be so cooperative, one would expect to see a few string states produced at the LHC. The leading contributions of Regge recurrences to certain processes at hadron colliders are universal. This is because the full-fledged string amplitudes which describe $2 \rightarrow 2$ parton scattering subprocesses involving four gauge bosons as well as those with two gauge bosons and two chiral matter fields are (to leading order in string coupling, but all orders in $\alpha^{\prime}$ ) independent of the compactification scheme. Only one assumption will be necessary in order to set up a solid framework: the string coupling must be small for the validity of perturbation theory in the computations of scattering amplitudes. In this case, black hole production and other strong gravity effects occur at energies above the string scale (see Appendix A); therefore, at least the few lowest Regge recurrences are available for examination, free from interference with some complex quantum gravitational phenomena. We discuss this next.

\section{Regge Recurrences}

The most direct way to compute the amplitude for the scattering of four gauge bosons is to consider the case of polarized particles because all nonvanishing contributions can be then generated from a single, maximally helicity violating (MHV), amplitude - the so-called partial MHV amplitude [97]. Assume that two vector bosons, with the momenta $k_{1}$ and $k_{2}$, in the $U(N)$ gauge group states corresponding to the generators $T^{a_{1}}$ and $T^{a_{2}}$ (here in the fundamental representation), carry negative helicities while the other two, with the momenta 
$k_{3}$ and $k_{4}$ and gauge group states $T^{a_{3}}$ and $T^{a_{4}}$, respectively, carry positive helicities. (All momenta are incoming.) Then the partial amplitude for such an MHV configuration is given by $[98,99]$

$$
\mathcal{A}\left(A_{1}^{-}, A_{2}^{-}, A_{3}^{+}, A_{4}^{+}\right)=4 g^{2} \operatorname{Tr}\left(T^{a_{1}} T^{a_{2}} T^{a_{3}} T^{a_{4}}\right) \frac{\langle 12\rangle^{4}}{\langle 12\rangle\langle 23\rangle\langle 34\rangle\langle 41\rangle} V\left(k_{1}, k_{2}, k_{3}, k_{4}\right),
$$

where $g$ is the $U(N)$ coupling constant, $\langle i j\rangle$ are the standard spinor products written in the notation of $[100,101]$, and the Veneziano form factor

$$
V\left(k_{1}, k_{2}, k_{3}, k_{4}\right)=V(s, t, u)=\frac{s u}{t M_{s}^{2}} B\left(-\frac{s}{M_{s}^{2}},-\frac{u}{M_{s}^{2}}\right)=\frac{\Gamma\left(1-s / M_{s}^{2}\right) \Gamma\left(1-u / M_{s}^{2}\right)}{\Gamma\left(1+t / M_{s}^{2}\right)}
$$

is the function of Mandelstam variables, $s=2 k_{1} k_{2}, t=2 k_{1} k_{3}, u=2 k_{1} k_{4} ; s+t+u=0$. (For simplicity we drop carets for the parton subprocess.) The physical content of the form factor becomes clear after using the well-known expansion in terms of s-channel resonances [102]

$$
B\left(-\frac{s}{M_{s}^{2}},-\frac{u}{M_{s}^{2}}\right)=-\sum_{n=0}^{\infty} \frac{M_{s}^{2-2 n}}{n !} \frac{1}{s-n M_{s}^{2}}\left[\prod_{J=1}^{n}\left(u+M_{s}^{2} J\right)\right],
$$

which exhibits s-channel poles associated to the propagation of virtual Regge excitations with masses $\sqrt{n} M_{s}$. Thus, near the $n$th level pole $\left(s \rightarrow n M_{s}^{2}\right)$,

$$
V(s, t, u) \approx \frac{1}{s-n M_{s}^{2}} \times \frac{M_{s}^{2-2 n}}{(n-1) !} \prod_{J=0}^{n-1}\left(u+M_{s}^{2} J\right)
$$

In specific amplitudes, the residues combine with the remaining kinematic factors, reflecting the spin content of particles exchanged in the $s$-channel, ranging from $J=0$ to $J=n+1$. (There are resonances in all the channels, that is, there are single particle poles in the $t$ and $u$ channels which would show up as bumps if $t$ or $u$ are positive. However, for physical scattering $t$ and $u$ are negative, so we do not see the bumps.) The low-energy expansion reads

$$
V(s, t, u) \approx 1-\frac{\pi^{2}}{6} \frac{s u}{M_{s}^{4}}-\zeta(3) \frac{s t u}{M_{s}^{6}}+\cdots
$$

Interestingly, because of the proximity of the 8 gluons and the photon on the color stack of D-branes, the gluon fusion into $\gamma+$ jet couples at tree level [103]. This implies that there is an order $g^{2}$ contribution in string theory, whereas this process is not occurring until order $g^{4}$ (loop level) in field theory. One can write down the total amplitude for this process projecting the gamma ray onto the hypercharge,

$$
\mathcal{M}(g g \longrightarrow \gamma g)=\cos \theta_{W} \mathcal{M}(g g \longrightarrow Y g)=\kappa \cos \theta_{W} \mathcal{M}(g g \longrightarrow C g)
$$


The $C-Y$ mixing coefficient evaluated at the scale for $U(N)$ unification $M_{S}$ follows from (4.4) and is given by

$$
\kappa=\frac{c_{3} g_{Y}}{g_{3}^{\prime}}=\frac{g_{Y}}{\sqrt{6} g_{3}} .
$$

It is quite small, around $\mathcal{K} \simeq 0.12$ for couplings evaluated at the $Z$ mass, which is modestly enhanced to $\kappa \simeq 0.14$ as a result of RG running of the couplings up to $\sim 5 \mathrm{TeV}$.

Consider the amplitude involving three $S U(N)$ gluons $g_{1}, g_{2}, g_{3}$ and one $U(1)$ gauge boson $\gamma_{4}$ associated to the same $U(N)$ stack:

$$
T^{a_{1}}=T^{a}, \quad T^{a_{2}}=T^{b}, \quad T^{a_{3}}=T^{c}, \quad T^{a_{4}}=Q \mathbb{I},
$$

where $\mathbb{I}$ is the $N \times N$ identity matrix and $Q$ is the $U(1)$ charge of the fundamental representation. The color factor is

$$
\operatorname{Tr}\left(T^{a_{1}} T^{a_{2}} T^{a_{3}} T^{a_{4}}\right)=Q\left(d^{a b c}+\frac{i}{4} f^{a b c}\right),
$$

where the totally symmetric symbol $d^{a b c}$ is the symmetrized trace while $f^{a b c}$ is the totally antisymmetric structure constant [100,101].

The full MHV amplitude can be obtained $[98,99]$ by summing the partial amplitudes (6.1) with the indices permuted in the following way:

$$
\mathcal{M}\left(g_{1}^{-}, g_{2}^{-}, g_{3}^{+}, \gamma_{4}^{+}\right)=4 g^{2}\langle 12\rangle^{4} \sum_{\sigma} \frac{\operatorname{Tr}\left(T^{a_{1_{\sigma}}} T^{a_{2_{\sigma}}} T^{a_{3_{\sigma}}} T^{a_{4}}\right) V\left(k_{1_{\sigma}}, k_{2_{\sigma}}, k_{3_{\sigma}}, k_{4}\right)}{\left\langle 1_{\sigma} 2_{\sigma}\right\rangle\left\langle 2_{\sigma} 3_{\sigma}\right\rangle\left\langle 3_{\sigma} 4\right\rangle\left\langle 41_{\sigma}\right\rangle}
$$

where the sum runs over all 6 permutations $\sigma$ of $\{1,2,3\}$ and $i_{\sigma} \equiv \sigma(i)$. Note that in the effective field theory of gauge bosons there are no Yang-Mills interactions that could generate this scattering process at the tree level. Indeed, $V=1$ at the leading order of (6.5) and the amplitude vanishes due to the following identity:

$$
\frac{1}{\langle 12\rangle\langle 23\rangle\langle 34\rangle\langle 41\rangle}+\frac{1}{\langle 23\rangle\langle 31\rangle\langle 14\rangle\langle 42\rangle}+\frac{1}{\langle 31\rangle\langle 12\rangle\langle 24\rangle\langle 43\rangle}=0 .
$$

Similarly, the antisymmetric part of the color factor (6.9) cancels out in the full amplitude (6.10). As a result, one obtains

$$
\mathcal{M}\left(g_{1}^{-}, g_{2}^{-}, g_{3}^{+}, \gamma_{4}^{+}\right)=8 Q d^{a b c} g^{2}\langle 12\rangle^{4}\left(\frac{\mu(s, t, u)}{\langle 12\rangle\langle 23\rangle\langle 34\rangle\langle 41\rangle}+\frac{\mu(s, u, t)}{\langle 12\rangle\langle 24\rangle\langle 13\rangle\langle 34\rangle}\right),
$$

where

$$
\mu(s, t, u)=\Gamma\left(1-\frac{u}{M_{s}^{2}}\right)\left(\frac{\Gamma\left(1-s / M_{s}^{2}\right)}{\Gamma\left(1+t / M_{s}^{2}\right)}-\frac{\Gamma\left(1-t / M_{s}^{2}\right)}{\Gamma\left(1+s / M_{s}^{2}\right)}\right) .
$$


All nonvanishing amplitudes can be obtained in a similar way. In particular,

$$
\mathcal{M}\left(g_{1}^{-}, g_{2}^{+}, g_{3}^{-}, \gamma_{4}^{+}\right)=8 Q d^{a b c} g^{2}\langle 13\rangle^{4}\left(\frac{\mu(t, s, u)}{\langle 13\rangle\langle 24\rangle\langle 14\rangle\langle 23\rangle}+\frac{\mu(t, u, s)}{\langle 13\rangle\langle 24\rangle\langle 12\rangle\langle 34\rangle}\right),
$$

and the remaining ones can be obtained either by appropriate permutations or by complex conjugation.

In order to obtain the cross section for the (unpolarized) partonic subprocess $g g \rightarrow$ $g \gamma$, we take the squared moduli of individual amplitudes, sum over final polarizations and colors, and average over initial polarizations and colors. As an example, the modulus square of the amplitude (6.10) is

$$
\left|\mathcal{M}\left(g_{1}^{-}, g_{2}^{-}, g_{3}^{+}, r_{4}^{+}\right)\right|^{2}=64 Q^{2} d^{a b c} d^{a b c} g^{4}\left|\frac{s \mu(s, t, u)}{u}+\frac{s \mu(s, u, t)}{t}\right|^{2}
$$

Taking into account all $4\left(N^{2}-1\right)^{2}$ possible initial polarization/color configurations and the formula [105]

$$
\sum_{a, b, c} d^{a b c} d^{a b c}=\frac{\left(N^{2}-1\right)\left(N^{2}-4\right)}{16 N}
$$

we obtain the average squared amplitude [103]:

$$
|\mathcal{M}(g g \longrightarrow g \gamma)|^{2}=g^{4} Q^{2} C(N)\left\{\left|\frac{s \mu(s, t, u)}{u}+\frac{s \mu(s, u, t)}{t}\right|^{2}+(s \longleftrightarrow t)+(s \longleftrightarrow u)\right\}
$$

where

$$
C(N)=\frac{2\left(N^{2}-4\right)}{N\left(N^{2}-1\right)}
$$

Before proceeding, we need to make sure of the value of $Q$. If we were considering the process $g g \rightarrow C g$, then $Q=\sqrt{1 / 6}$ due to the normalization condition (2.21). However, for $g g \rightarrow \gamma g$ there are two additional projections given in (6.6): from $C_{\mu}$ to the hypercharge boson $Y_{\mu}$, yielding a mixing factor $\kappa$ and from $Y_{\mu}$ onto a photon, providing an additional factor $\cos \theta_{W}$. This gives

$$
Q=\sqrt{\frac{1}{6}} \kappa \cos \theta_{W}
$$


The two most interesting energy regimes of $g g \rightarrow g r$ scattering are far below the string mass scale $M_{s}$ and near the threshold for the production of massive string excitations. At low energies, (6.17) becomes

$$
|\mathcal{M}(g g \longrightarrow g \gamma)|^{2} \approx g^{4} Q^{2} C(N) \frac{\pi^{4}}{4 M_{s}^{8}}\left(s^{4}+t^{4}+u^{4}\right), \quad\left(s, t, u \ll M_{s}^{2}\right) .
$$

The absence of massless poles, at $s=0$, and so forth, translated into the terms of effective field theory, confirms that there are no exchanges of massless particles contributing to this process. On the other hand, near the string threshold $s \approx M_{s}^{2}$,

$$
|\mathcal{M}(g g \longrightarrow g \gamma)|^{2} \approx 4 g^{4} Q^{2} C(N) \frac{M_{s}^{8}+t^{4}+u^{4}}{M_{s}^{4}\left(s-M_{s}^{2}\right)^{2}}, \quad\left(s \approx M_{s}^{2}\right)
$$

see Appendix B for details.

The general form of (6.10) for any given four external gauge bosons reads

$$
\begin{aligned}
\mathcal{M}\left(A_{1}^{-}, A_{2}^{-}, A_{3}^{+}, A_{4}^{+}\right)=4 g^{2}\langle 12\rangle^{4}[ & \frac{V_{t}}{\langle 12\rangle\langle 23\rangle\langle 34\rangle\langle 41\rangle} \operatorname{Tr}\left(T^{a_{1}} T^{a_{2}} T^{a_{3}} T^{a_{4}}+T^{a_{2}} T^{a_{1}} T^{a_{4}} T^{a_{3}}\right) \\
& +\frac{V_{u}}{\langle 13\rangle\langle 34\rangle\langle 42\rangle\langle 21\rangle} \operatorname{Tr}\left(T^{a_{2}} T^{a_{1}} T^{a_{3}} T^{a_{4}}+T^{a_{1}} T^{a_{2}} T^{a_{4}} T^{a_{3}}\right) \\
& \left.+\frac{V_{s}}{\langle 14\rangle\langle 42\rangle\langle 23\rangle\langle 31\rangle} \operatorname{Tr}\left(T^{a_{1}} T^{a_{3}} T^{a_{2}} T^{a_{4}}+T^{a_{3}} T^{a_{1}} T^{a_{4}} T^{a_{2}}\right)\right],
\end{aligned}
$$

where

$$
V_{t}=V(s, t, u), \quad V_{u}=V(t, u, s), \quad V_{s}=V(u, s, t) .
$$

The modulus square of the four-gluon amplitude, summed over final polarizations and colors and averaged over all $4\left(N^{2}-1\right)^{2}$ possible initial polarization/color configurations, follows from (6.22) and is given by [106]

$$
\begin{aligned}
|\mathcal{M}(g g \longrightarrow g g)|^{2}= & g^{4}\left(\frac{1}{s^{2}}+\frac{1}{t^{2}}+\frac{1}{u^{2}}\right) \\
& \times\left[\frac{2 N^{2}}{N^{2}-1}\left(s^{2} V_{s}^{2}+t^{2} V_{t}^{2}+u^{2} V_{u}^{2}\right)+\frac{4\left(3-N^{2}\right)}{N^{2}\left(N^{2}-1\right)}\left(s V_{s}+t V_{t}+u V_{u}\right)^{2}\right]
\end{aligned}
$$

The amplitudes involving two gluons and two quarks are also independent of the details of the compactification, such as the configuration of branes, the geometry of the extra dimensions, and whether SUSY is broken or not. This model independence makes it possible to compute all the string corrections to dijet signals at the LHC. The corresponding 


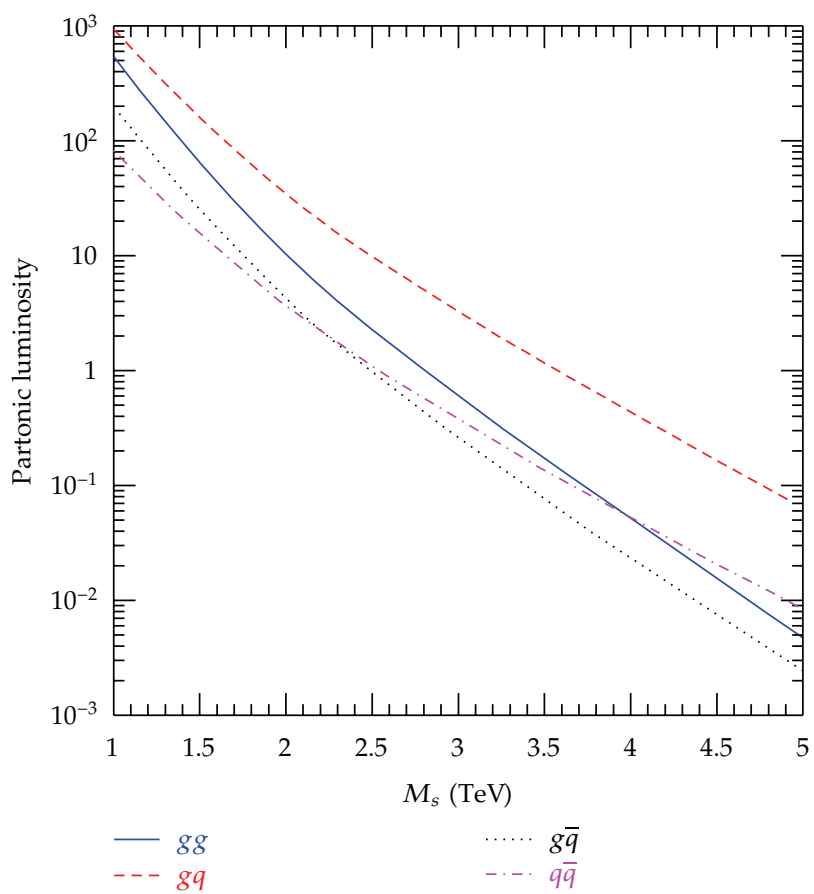

Figure 5: Relative contributions of initial state partons $(i j=g g, g q, g \bar{q}$, and $q \bar{q})$ to $\int_{\tau_{0}}^{1} f_{i}\left(x_{a}, Q\right) f_{j}\left(\tau_{0} /\right.$ $\left.x_{a}, Q\right) d x_{a} / x_{a}$, with varying string scale. From [104].

$2 \rightarrow 2$ scattering amplitudes, computed at the leading order in string perturbation theory, are collected in [106]. The average square amplitudes are given by the following:

$$
\begin{gathered}
|\mathcal{M}(g g \longrightarrow q \bar{q})|^{2}=g^{4} N_{f} \frac{t^{2}+u^{2}}{s^{2}}\left[\frac{1}{2 N} \frac{1}{u t}\left(t V_{t}+u V_{u}\right)^{2}-\frac{N}{N^{2}-1} V_{t} V_{u}\right], \\
|\mathcal{M}(q \bar{q} \longrightarrow g g)|^{2}=g^{4} \frac{t^{2}+u^{2}}{s^{2}}\left[\frac{\left(N^{2}-1\right)^{2}}{2 N^{3}} \frac{1}{u t}\left(t V_{t}+u V_{u}\right)^{2}-\frac{N^{2}-1}{N} V_{t} V_{u}\right] \\
|\mathcal{M}(q g \longrightarrow q g)|^{2}=g^{4} \frac{s^{2}+u^{2}}{t^{2}}\left[V_{s} V_{u}-\frac{N^{2}-1}{2 N^{2}} \frac{1}{s u}\left(s V_{s}+u V_{u}\right)^{2}\right]
\end{gathered}
$$

The amplitudes for the four-fermion processes like quark-antiquark scattering are more complicated because the respective form factors describe not only the exchanges of Regge states but also of heavy Kaluza-Klein and winding states with a model-dependent spectrum determined by the geometry of extra dimensions. Fortunately, they are suppressed, for two reasons. First, the QCD SU(3) color group factors favor gluons over quarks in the initial state. Second, the parton luminosities in proton-proton collisions at the LHC, at the parton center of mass energies above $1 \mathrm{TeV}$, are significantly lower for quark-antiquark subprocesses than for gluon-gluon and gluon-quark, see Figure 5. The collisions of valence quarks occur at higher luminosity; however, there are no Regge recurrences appearing in the s-channel of quark-quark scattering [106]. 
In the following, we isolate the contribution from the first resonant state in (6.24)(6.27). For partonic center of mass energies $\sqrt{s}<M_{s}$, contributions from the Veneziano functions are strongly suppressed, as $\sim\left(\sqrt{s} / M_{s}\right)^{8}$, over standard model processes, see (6.20). (Corrections to SM processes at $\sqrt{s} \ll M_{s}$ are of order $\left(\sqrt{s} / M_{s}\right)^{4}$, see (6.5)). In order to factorize amplitudes on the poles due to the lowest massive string states, it is sufficient to consider $s=M_{s}^{2}$. In this limit, $V_{s}$ is regular while

$$
V_{t} \longrightarrow \frac{u}{s-M_{s}^{2}}, \quad V_{u} \longrightarrow \frac{t}{s-M_{s}^{2}}
$$

Thus the s-channel pole term of the average square amplitude (6.24) can be rewritten as

$$
|\mathcal{M}(g g \longrightarrow g g)|^{2}=2 \frac{g^{4}}{M_{s}^{4}}\left(\frac{N^{2}-4+\left(12 / N^{2}\right)}{N^{2}-1}\right) \frac{M_{s}^{8}+t^{4}+u^{4}}{\left(s-M_{s}^{2}\right)^{2}}
$$

Note that the contributions of single poles to the cross section are antisymmetric about the position of the resonance and vanish in any integration over the resonance. (As an illustration, consider the amplitude $a+b / D$ in the vicinity of the pole, where $a$ and $b$ are real, $D=x+i \epsilon, x=s-M_{s}^{2}$, and $\epsilon=\Gamma M_{s}$. Then, since $\operatorname{Re}(1 / D)=x /|D|^{2}$, the cross section becomes $\sigma \propto a^{2}+b^{2} /|D|^{2}+2 a b x /|D|^{2} \simeq a^{2}+b^{2} \pi \delta(x) / \epsilon+2 a b \pi x \delta(x) / \epsilon$. Integrating over the width of the resonance, one obtains $a^{2} \epsilon+b^{2} \pi / \epsilon \simeq b \pi$, because $b \propto \epsilon, a \propto g^{2}$, and $\epsilon \propto g^{2}$.) Before proceeding, we pause to present our notation. The first Regge excitations of the gluon $g$, the color singlet $C$, and quarks $q$ will be denoted by $g^{*}, C^{*}, q^{*}$, respectively. Recall that $C_{\mu}$ has an anomalous mass in general lower than the string scale by an order of magnitude. If that is the case, and if the mass of the $C^{*}$ is composed (approximately) of the anomalous mass of the $C_{\mu}$ and $M_{s}$ added in quadrature, we would expect only a minor error in our results by taking the $C^{*}$ to be degenerate with the other resonances. The singularity at $s=M_{s}^{2}$ needs softening to a Breit-Wigner form, reflecting the finite decay widths of resonances propagating in the $s$ channel. Due to averaging over initial polarizations, (6.29) contains additive contributions from both spin $J=0$ and spin $J=2 U(3)$ bosonic Regge excitations $\left(g^{*}\right.$ and $\left.C^{*}\right)$, created by the incident gluons in the helicity configurations $( \pm \pm)$ and $( \pm \mp)$, respectively. The $M_{s}^{8}$ term in (6.29) originates from $J=0$, and the $t^{4}+u^{4}$ piece reflects $J=2$ activity. Since the resonance widths depend on the spin and on the identity of the intermediate state $\left(g^{*}, C^{*}\right)$, the pole term (6.29) should be smeared as [107]

$$
\begin{aligned}
|\mathcal{M}(g g \longrightarrow g g)|^{2}= & 2 \frac{g^{4}}{M_{s}^{4}}\left(\frac{N^{2}-4+\left(12 / N^{2}\right)}{N^{2}-1}\right) \\
& \times\left\{W_{g^{*}}^{g g \rightarrow g g}\left[\frac{M_{s}^{8}}{\left(s-M_{s}^{2}\right)^{2}+\left(\Gamma_{g^{*}}^{J=0} M_{s}\right)^{2}}+\frac{t^{4}+u^{4}}{\left(s-M_{s}^{2}\right)^{2}+\left(\Gamma_{g^{*}}^{J=2} M_{s}\right)^{2}}\right]\right. \\
& \left.+W_{C^{O *}}^{g g \rightarrow g g}\left[\frac{M_{s}^{8}}{\left(s-M_{s}^{2}\right)^{2}+\left(\Gamma_{C^{*}}^{J=0} M_{s}\right)^{2}}+\frac{t^{4}+u^{4}}{\left(s-M_{s}^{2}\right)^{2}+\left(\Gamma_{C^{*}}^{J=2} M_{s}\right)^{2}}\right]\right\},
\end{aligned}
$$


where $\Gamma_{g^{*}}^{J=0}=75\left(M_{s} / \mathrm{TeV}\right) \mathrm{GeV}, \Gamma_{C^{*}}^{J=0}=150\left(M_{s} / \mathrm{TeV}\right) \mathrm{GeV}, \Gamma_{g^{*}}^{J=2}=45\left(M_{s} / \mathrm{TeV}\right) \mathrm{GeV}$, and $\Gamma_{C^{*}}^{J=2}=75\left(M_{s} / \mathrm{TeV}\right) \mathrm{GeV}$ are the total decay widths for intermediate states $g^{*}$ and $C^{*}$, with angular momentum $J$ [108]. The associated weights of these intermediate states are given in terms of the probabilities for the various entrance and exit channels

$$
\begin{aligned}
\frac{N^{2}-4+12 / N^{2}}{N^{2}-1} & =\frac{16}{\left(N^{2}-1\right)^{2}}\left[\left(N^{2}-1\right)\left(\frac{N^{2}-4}{4 N}\right)^{2}+\left(\frac{N^{2}-1}{2 N}\right)^{2}\right] \\
& \propto \frac{16}{\left(N^{2}-1\right)^{2}}\left[\left(N^{2}-1\right)\left(\Gamma_{g^{*} \rightarrow g g}\right)^{2}+\left(\Gamma_{C^{*} \rightarrow g g}\right)^{2}\right],
\end{aligned}
$$

yielding

$$
\begin{aligned}
& W_{g^{*}}^{g g \rightarrow g g}=\frac{8\left(\Gamma_{g^{*} \rightarrow g g}\right)^{2}}{8\left(\Gamma_{g^{*} \rightarrow g g}\right)^{2}+\left(\Gamma_{C^{*} \rightarrow g g}\right)^{2}}=0.44, \\
& W_{C^{*}}^{g g \rightarrow g g}=\frac{\left(\Gamma_{C^{*} \rightarrow g g}\right)^{2}}{8\left(\Gamma_{g^{*} \rightarrow g g}\right)^{2}+\left(\Gamma_{C^{*} \rightarrow g g}\right)^{2}}=0.56 .
\end{aligned}
$$

A similar calculation transforms (6.25) near the pole into

$$
\begin{aligned}
|\mathcal{M}(g g \longrightarrow q \bar{q})|^{2}= & \frac{g^{4}}{M_{s}^{4}} N_{f}\left(\frac{N^{2}-2}{N\left(N^{2}-1\right)}\right) \\
& \times\left[W_{g^{*}}^{g g \rightarrow q \bar{q}} \frac{u t\left(u^{2}+t^{2}\right)}{\left(s-M_{s}^{2}\right)^{2}+\left(\Gamma_{g^{*}}^{J=2} M_{s}\right)^{2}}+W_{C^{*}}^{g g \rightarrow q \bar{q}} \frac{u t\left(u^{2}+t^{2}\right)}{\left(s-M_{s}^{2}\right)^{2}+\left(\Gamma_{C^{*}}^{J=2} M_{s}\right)^{2}}\right],
\end{aligned}
$$

where

$$
\begin{aligned}
& W_{g^{*}}^{g g \rightarrow q \bar{q}}=W_{g^{*}}^{q \bar{q} \rightarrow g g}=\frac{8 \Gamma_{g^{*} \rightarrow g g} \Gamma_{g^{*} \rightarrow q \bar{q}}}{8 \Gamma_{g^{*} \rightarrow g g} \Gamma_{g^{*} \rightarrow q \bar{q}}+\Gamma_{C^{*} \rightarrow g g} \Gamma_{C^{*} \rightarrow q \bar{q}}}=0.71, \\
& W_{C^{*}}^{g g \rightarrow q \bar{q}}=W_{C^{*}}^{q \bar{q} \rightarrow g g}=\frac{\Gamma_{C^{*} \rightarrow g g} \Gamma_{C^{*} \rightarrow q \bar{q}}}{8 \Gamma_{g^{*} \rightarrow g g} \Gamma_{g^{*} \rightarrow q \bar{q}}+\Gamma_{C^{*} \rightarrow g g} \Gamma_{C^{*} \rightarrow q \bar{q}}}=0.29 .
\end{aligned}
$$


Near the $s$ pole, (6.26) becomes

$$
\begin{aligned}
|\mathcal{M}(q \bar{q} \longrightarrow g g)|^{2}= & \frac{g^{4}}{M_{s}^{4}}\left(\frac{\left(N^{2}-2\right)\left(N^{2}-1\right)}{N^{3}}\right) \\
& \times\left[W_{g^{*}}^{q \bar{q} \rightarrow g g} \frac{u t\left(u^{2}+t^{2}\right)}{\left(s-M_{s}^{2}\right)^{2}+\left(\Gamma_{g^{*}}^{J=2} M_{s}\right)^{2}}+W_{C^{*}}^{q \bar{q} \rightarrow g g} \frac{u t\left(u^{2}+t^{2}\right)}{\left(s-M_{s}^{2}\right)^{2}+\left(\Gamma_{C^{*}}^{J=2} M_{s}\right)^{2}}\right],
\end{aligned}
$$

whereas (6.27) can be rewritten as

$$
\begin{aligned}
|\mathcal{M}(q g \longrightarrow q g)|^{2}= & -\frac{g^{4}}{M_{s}^{2}}\left(\frac{N^{2}-1}{2 N^{2}}\right) \\
& \times\left[\frac{M_{s}^{4} u}{\left(s-M_{s}^{2}\right)^{2}+\left(\Gamma_{q^{*}}^{J=1 / 2} M_{s}\right)^{2}}+\frac{u^{3}}{\left(s-M_{s}^{2}\right)^{2}+\left(\Gamma_{q^{*}}^{J=3 / 2} M_{s}\right)^{2}}\right] .
\end{aligned}
$$

The total decay widths for the $q^{*}$ excitation are $\Gamma_{q^{*}}^{J=1 / 2}=\Gamma_{q^{*}}^{J=3 / 2}=37\left(M_{s} / \mathrm{TeV}\right) \mathrm{GeV}$ [108]. Superscripts $J=2$ are understood to be inserted on all the $\Gamma^{\prime}$ s in (6.32) and (6.34). Equation (6.30) reflects the fact that weights for $J=0$ and $J=2$ are the same [108].

The $s$-channel poles near the second Regge resonance can be approximated by expanding the Veneziano formfactor $V_{t}$ around $s=2 M_{s}^{2}$,

$$
V(s, t, u) \approx \frac{u\left(u+M_{s}^{2}\right)}{M_{s}^{2}\left(s-2 M_{s}^{2}\right)}
$$

The associated scattering amplitudes and decay widths of the $n=2$ string resonances are collected in [109]. Generally, the width of the Regge excitations will grow at least linearly with energy, whereas the spacing between levels will decrease with energy. This implies an upper limit on the domain of validity for this phenomenological approach [110]. In particular, for a resonance $R$ of mass $M$, the total width is given by

$$
\Gamma_{\mathrm{tot}} \sim \frac{g^{2}}{4 \pi} \mathcal{C} \frac{M}{4}
$$

where $C>1$ because of the growing multiplicity of decay modes $[108,109]$. On the other hand, since $\Delta\left(M^{2}\right)=M_{s}^{2}$, the level spacing at mass $M$ is $\Delta M \sim M_{s}^{2} /(2 M)$; thus,

$$
\frac{\Gamma_{\text {tot }}}{\Delta M} \sim \frac{g^{2}}{8 \pi} C\left(\frac{M}{M_{s}}\right)^{2}=\frac{g^{2}}{8 \pi} C n<1
$$


For excitation of the resonance $R$ via $a+b \rightarrow R$, the assumption $\Gamma_{\text {tot }}(R) \sim \Gamma(R \rightarrow a b)$ (which underestimates the real width) yields a perturbative regime for $n \lesssim 40$. This is to be compared with the $n \sim 10^{4}$ levels of the string needed for black hole production (see Appendix A).

Given the particular nature of the process we are considering, the production of a $\mathrm{TeV}$-scale resonance, and its subsequent 2-body decay, several observables at the LHC are available. Most apparently, one would hope that the resonance would be visible in data binned according to the dijet invariant mass $M$, after setting cuts on the different jet rapidities, $\left|y_{1}\right|,\left|y_{2}\right| \leq y_{\max }$ and transverse momenta $p_{T}^{1,2}>50 \mathrm{GeV}$. With the definitions $Y \equiv(1 / 2)\left(y_{1}+y_{2}\right)$ and $y \equiv(1 / 2)\left(y_{1}-y_{2}\right)$, the cross section per interval of $M$ for $p p \rightarrow$ dijet is given by [111]

$$
\begin{aligned}
\frac{d \sigma}{d M}=M \tau \sum_{i j k l} & {\left[\left.\int_{-Y_{\max }}^{0} d Y f_{i}\left(x_{a}, M\right) f_{j}\left(x_{b}, M\right) \int_{-\left(y_{\max }+Y\right)}^{y_{\max }+Y} d y \frac{d \sigma}{d \hat{t}}\right|_{i j \rightarrow k l} \frac{1}{\cosh ^{2} y}\right.} \\
& \left.+\left.\int_{0}^{Y_{\max }} d Y f_{i}\left(x_{a}, M\right) f_{j}\left(x_{b}, M\right) \int_{-\left(y_{\max }-Y\right)}^{y_{\max }-Y} d y \frac{d \sigma}{d \widehat{t}}\right|_{i j \rightarrow k l} \frac{1}{\cosh ^{2} y}\right],
\end{aligned}
$$

where $f(x, M)$ 's are parton distribution functions (we use CTEQ6 [63]), $\tau=M^{2} / s, x_{a}=$ $\sqrt{\tau} e^{Y}, x_{b}=\sqrt{\tau} e^{-Y}$, and

$$
|\mathcal{M}(i j \longrightarrow k l)|^{2}=\left.16 \pi \widehat{s}^{2} \frac{d \sigma}{d \hat{t}}\right|_{i j \rightarrow k l}
$$

We reinstate the caret notation $(\widehat{s}, \widehat{t}, \widehat{u})$ to specify partonic subprocesses. The $Y$ integration range in (6.40), $Y_{\max }=\min \left\{\ln (1 / \sqrt{\tau}), y_{\max }\right\}$, comes from requiring $x_{a}, x_{b}<1$ together with the rapidity cuts $y_{\min }<\left|y_{1}\right|,\left|y_{2}\right|<y_{\max }$. The kinematics of the scattering also provides the relation $M=2 p_{T} \cosh y$, which when combined with $p_{T}=M / 2 \sin \theta^{*}=M / 2 \sqrt{1-\cos ^{2} \theta^{*}}$, yields $\cosh y=\left(1-\cos ^{2} \theta^{*}\right)^{-1 / 2}$, where $\theta^{*}$ is the center-of-mass scattering angle. Finally, the Mandelstam invariants occurring in the cross section are given by $\widehat{s}=M^{2}, \widehat{t}=$ $-(1 / 2) M^{2} e^{-y} / \cosh y$, and $\hat{u}=-(1 / 2) M^{2} e^{+y} / \cosh y$. In what follows we set $N=3$ and $N_{f}=6$.

The CMS collaboration has searched for such narrow resonances in their dijet mass spectrum using data from $p p$ collisions at $\sqrt{s}=7 \mathrm{TeV}[112,113]$. After operating for only few months, with merely 2.9 inverse picobarns of integrated luminosity, the LHC CMS experiment has ruled out $M_{s}<2.5 \mathrm{TeV}$. The LHC7 has recently delivered an integrated luminosity in excess of $1 \mathrm{fb}^{-1}$. This extends considerably the search territory for new physics in events containing dijets. The new data exclude string resonances with $M_{s}<4 \mathrm{TeV}$ [65]. In fact, the LHC has the capacity of discovering strongly interacting resonances via dijet final states in practically all range up to $(1 / 2) \sqrt{s}_{\text {LHC }}$. We discuss this next.

Standard bump-hunting methods, such as calculating cumulative cross sections

$$
\sigma\left(M_{0}\right)=\int_{M_{0}}^{\infty} \frac{d \sigma}{d M} d M
$$

and searching for regions with significant deviations from the QCD background, may allow to find an interval of $M$ suspected of containing a bump. With the establishment of such a region, one may calculate a signal-to-noise ratio, with the signal rate estimated in the invariant mass window $\left[M_{s}-2 \Gamma, M_{s}+2 \Gamma\right]$. To accommodate the minimal acceptance cuts 
on dijets from LHC experiments [114], an additional kinematic cut, $\left|y_{\max }\right|<1.0$, has been included in the calculation. The noise is defined as the square root of the number of QCD background events in the same dijet mass interval for the same integrated luminosity. Our significant results are encapsuled in Figure 6, where we show the signal-to-noise ratio as a function of the string scale. It is remarkable that within one to two years of data collection with LHC14, string scales as large as $6.8 \mathrm{TeV}$ are open to discovery at the $\geq 5 \sigma$ level. Once more, we stress that these results contain no unknown parameters. They depend only on the Dbrane construct for the SM and are independent of compactification details. (The only remnant of the compactification is the relation between the Yang-Mills coupling and the string coupling. We take this relation to reduce to field theoretical results in the case where they exist, for example, $g g \rightarrow g g$. Then, because of the require correspondence with field theory, the phenomenological results are independent of the compactification of the transverse space. However, a different phenomenology would result as a consequence of warping one [115] or more parallel dimensions [116-119]).

Although the expected discovery reach would not be as high as that for dijets, the measurement of $p p \rightarrow \gamma+$ jet can potentially provide an interesting corroboration for the stringy origin for new physics manifest as a resonant structure in LHC data. The Breit-Wigner form for gluon fusion into $\gamma+$ jet follows from (6.21) and is given by

$$
|\mathcal{M}(g g \longrightarrow g \gamma)|^{2} \simeq \frac{5 g_{3}^{4} Q^{2}}{3 M_{s}^{4}}\left[\frac{M_{s}^{8}}{\left(\widehat{s}-M_{s}^{2}\right)^{2}+\left(\Gamma_{g^{*}}^{J=0} M_{s}\right)^{2}}+\frac{\widehat{t}^{4}+\widehat{u}^{4}}{\left(\widehat{s}-M_{s}^{2}\right)^{2}+\left(\Gamma_{g^{*}}^{J=2} M_{s}\right)^{2}}\right]
$$

From Figure 5 we see that the dominant $s$-channel pole term of the average square amplitude contributing to $p p \rightarrow \gamma+$ jet is [120]

$$
|\mathcal{M}(q g \longrightarrow q r)|^{2}=-\frac{g_{3}^{4} Q^{2}}{3 M_{s}^{2}}\left[\frac{M_{s}^{4} \widehat{u}}{\left(\widehat{s}-M_{s}^{2}\right)^{2}+\left(\Gamma_{q^{*}}^{J=1 / 2} M_{s}\right)^{2}}+\frac{\widehat{u}^{3}}{\left(\widehat{s}-M_{s}^{2}\right)^{2}+\left(\Gamma_{q^{*}}^{J=3 / 2} M_{s}\right)^{2}}\right]
$$

We duplicate the calculation of the signal-to-noise ratio substituting in (6.40) $d \sigma / d \widehat{t}_{i j \rightarrow k l}$ by $d \sigma / d \widehat{t}_{i j \rightarrow k \gamma}$. For photons, we set $y_{\max }<2.4[121,122]$. To minimize misidentification with a high- $p_{T} \pi^{0}$, isolation cuts must be imposed on the photon, and to trigger on the desired channel, the hadronic jet must be identified. A detailed study of the CMS potential for isolation of prompt- $\gamma$ 's has been carried out [123], using GEANT4 simulations of $\gamma+$ jet events generated with Pythia. This analysis (which also includes $\gamma^{\prime}$ s produced in the decays of $\eta, K_{s}^{0}, \omega^{0}$, and bremsstrahlung photons emerging from high- $p_{T}$ jets) suggests

$$
\beta=\frac{\text { background due to misidentified } \pi^{0} \text { after isolation cuts }}{\text { QCD background from direct photon production }}+1 \simeq 2 \text {. }
$$

Therefore, in our numerical calculation we assume the noise is increased by a factor of $\sqrt{\beta}$, over the direct photon QCD contribution. The signal used to obtain the results displayed in Figure 6 includes the parton subprocesses $g g \rightarrow g r$ (which does not exist at tree level 


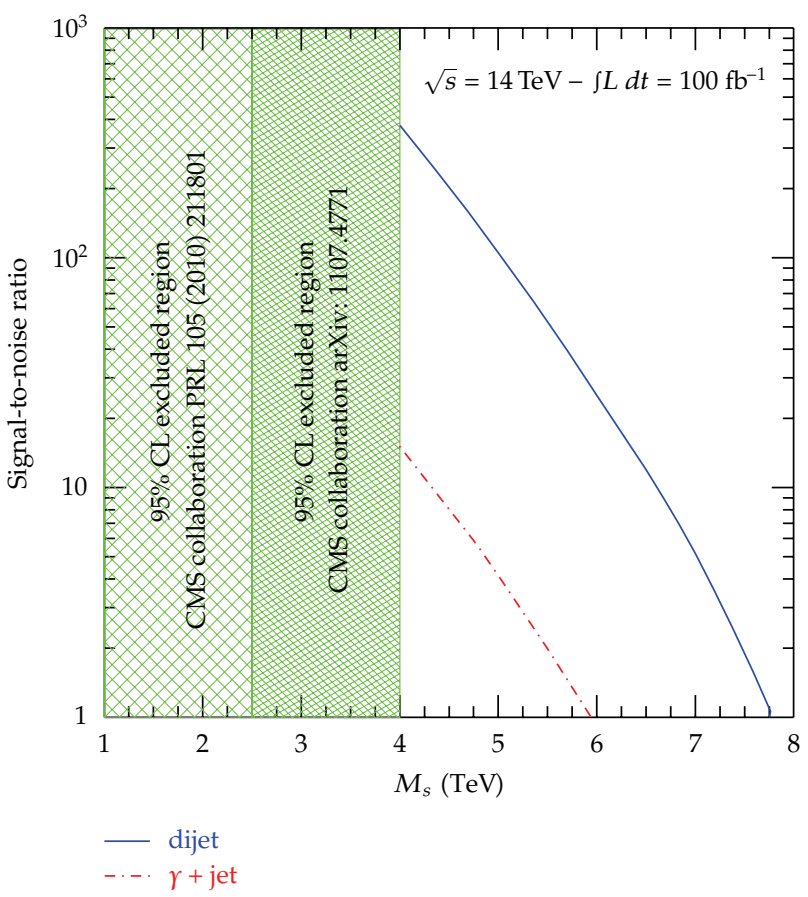

Figure 6: Signal-to-noise ratio of $p p \rightarrow$ dijet and $p p \rightarrow \gamma+$ jet, for $\sqrt{s}=14 \mathrm{TeV}, \mathcal{\perp}=100 \mathrm{fb}^{-1}$, and $\kappa^{2} \simeq 0.02$. The approximate equality of the background due to misidentified $\pi^{0 \prime}$ s and the QCD background, across a range of large $p_{T}^{\gamma}$, is maintained as an approximate equality over a range of $\gamma$-jet invariant masses with the rapidity cuts imposed $\left(\left|y_{\max }^{j}\right|<1.0\right.$ and $\left.\left|y_{\max }^{\gamma}\right|<2.4\right)$. The $95 \%$ CL lower limits on the string scale recently reported by the CMS collaboration are also shown.

in QCD), $q g \rightarrow q r, \bar{q} g \rightarrow \bar{q} r$, and $q \bar{q} \rightarrow g r$. All except the first have been calculated in QCD and constitute the SM background. For string scales as high as $5.0 \mathrm{TeV}$, observations of resonant structures in $p p \rightarrow \gamma+$ jet can provide interesting corroboration for stringy physics at the TeV-scale.

Events with a single jet plus missing energy $\left(E_{T}^{\prime}\right)$ with balancing transverse momenta (so-called "monojets") are incisive probes of new physics. As in the SM, the source of this topology is $i j \rightarrow k Z$ followed by $Z \rightarrow v \bar{v}$. Both in the SM and string theory, the cross section for this process is of order $g^{4}$. Virtual KK graviton emission $(i j \rightarrow k G)$ involves emission of closed strings, resulting in an additional suppression of order $g^{2}$ compared to $Z$ emission. A careful discussion of this suppression is given in [124]. Ignoring the $Z$-mass (i.e., keeping only transverse $Z$ 's), the Regge contribution to $p p \rightarrow Z+$ jet is suppressed relative to the $p p \rightarrow \gamma+$ jet by a factor of $\tan ^{2} \theta_{W}=0.29$ [125].

We now turn to the analysis of the dijet angular distributions. QCD partonparton cross sections are dominated by $t$-channel exchanges that produce dijet angular distributions which peak at small center of mass scattering angles. In contrast, nonstandard contact interactions or excitations of resonances result in a more isotropic distribution. In terms of rapidity variables for standard transverse momentum cuts, dijets resulting from QCD processes will preferentially populate the large rapidity region, while the new processes 
generate events more uniformly distributed in the entire rapidity region. To analyze the details of the rapidity space, the $\mathrm{D} \varnothing$ collaboration [126] introduced a new parameter:

$$
R=\frac{d \sigma /\left.d M\right|_{\left(\left|y_{1}\right|,\left|y_{2}\right|<0.5\right)}}{d \sigma /\left.d M\right|_{\left(0.5<\left|y_{1}\right|,\left|y_{2}\right|<1.0\right)}},
$$

the ratio of the number of events, in a given dijet mass bin, for both rapidities $\left|y_{1}\right|,\left|y_{2}\right|<0.5$ and both rapidities $0.5<\left|y_{1}\right|,\left|y_{2}\right|<1$.0. In Figure 7 we compare the results from a full CMS detector simulation of the ratio $R$, with predictions from LO QCD and model-independent contributions to the $q^{*}, g^{*}$, and $C^{*}$ excitations. (An illustration of the use of this parameter in a heuristic model where SM amplitudes are modified by a Veneziano formfactor has been presented [127].) The synthetic population was generated with Pythia, passed through the full CMS detector simulation and reconstructed with the ORCA reconstruction package [128]. For an integrated luminosity of $10 \mathrm{fb}^{-1}$, the LO QCD contributions with $\alpha_{s}=g_{3}^{2} / 4 \pi=0.1$ (corresponding to running scale $\Lambda \approx M_{s}$ ) are within statistical fluctuations of the full CMS detector simulation. (Note that the string scale is an optimal choice of the running scale which should normally minimize the role of higher loop corrections). Since one of the purposes of utilizing NLO calculations is to fix the choice of the running coupling, we take this agreement as rationale to omit loops in QCD and in string theory. It is clear from Figure 7 that incorporating NLO calculation of the background and the signal would not significantly change the large deviation of the string contribution from the QCD background. String scales $\sim 5 \mathrm{TeV}$ can be probed with $10 \mathrm{fb}^{-1}$ of LHC14 data collection. Because of background reduction by optimized rapidity cuts, the $R$ parameter can (in principle) extend the LHC discovery reach of Regge excitations.

In closing, we note that for $e^{+} e^{-}$colliders string theory predicts the precise value, equal to 1/3, of the relative weight of spin 2 and spin 1 contributions [129]. This yields a dimuon angular distribution with a pronounced forward-backward asymmetry, which could help distinguishing between low mass strings and other beyond SM scenarios.

\section{Conclusions}

We have considered a low-mass string compactification in which the SM gauge multiplets originate in open strings ending on D-branes, with gauge bosons due to strings attached to stacks of D-branes and chiral matter due to strings stretching between intersecting D-branes. For the nonabelian SU(3) group, the D-brane construct requires the existence of an additional $U(1)$ gauge boson coupled to baryon number. In this framework, $U(1)$ and $S U(3)$ appear as subgroups of $U(3)$. In addition, our minimal model contains three other stacks of D-branes to accommodate the electroweak $S p(1)$ left and $U(1)$ fields attached to the lepton D-brane and to the right D-brane. One linear combination of the three $U(1)$ gauge bosons is identified as the hypercharge $Y$ field, coupled to the anomaly free hypercharge current. The two remaining linear combinations $\left(Y^{\prime}, Y^{\prime \prime}\right)$ of the three $U(1)^{\prime}$ 's, which can be naturally associated with $B$ and $B-L$, grow masses. After electroweak breaking, mixing with the third component of isospin results in the three observable gauge bosons, with small mixing $Z^{\prime} \simeq Y^{\prime}, Z^{\prime \prime} \simeq Y^{\prime \prime}$.

In our phenomenological discussion about the possible discovery of massive $Z^{\prime}$-gauge bosons, we have taken as a benchmark scenario the dijet plus $W$ signal, recently observed by the CDF collaboration at Tevatron. For a fixed $M_{Z^{\prime}} \simeq 150 \mathrm{GeV}$, the model is quite constrained. Fine tuning its free parameters is just sufficient to simultaneously ensure a small $Z-Z$ ' mixing 


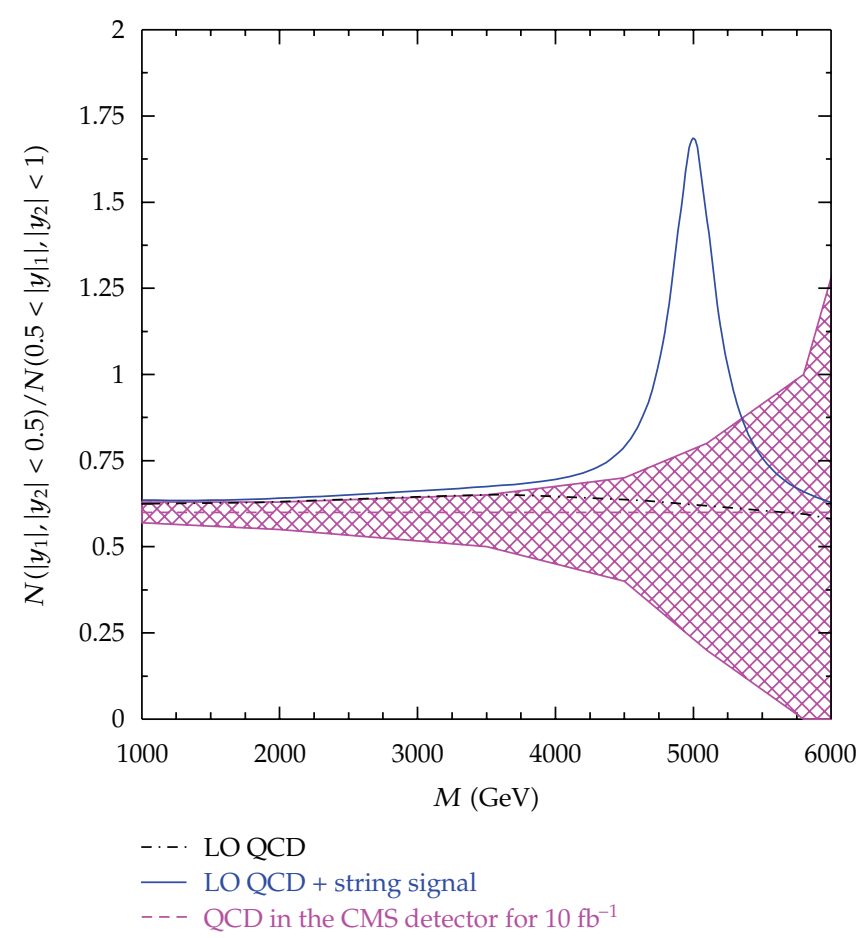

Figure 7: For a luminosity of $10 \mathrm{fb}^{-1}$, the expected value (dashed line) and statistical error (shaded region) of the dijet ratio of QCD in the CMS detector are compared with LO QCD (dot-dashed line) and LO QCD plus lowest massive string excitation at a scale $M_{s}=5 \mathrm{TeV}$. From [107].

in accord with the stringent LEP data on the $Z$ mass, very small (less than $1 \%$ ) branching ratio into leptons, and a large hierarchy between $Z^{\prime \prime}$ and $Z^{\prime}$ masses.

If the CDF anomaly does not survive additional scrutiny (as indicated by the more recent $\mathrm{D} \varnothing$ results), the analysis presented here can be directly applied to the higher energy realm, with a view toward identifying the precise makeup of the various abelian sectors, and pursuing with strong confidence a signal at LHC for Regge excitations of the string.

In D-brane constructions, the full-fledged string amplitudes supplying the dominant contributions to $p p$ scattering cross sections are completely independent of the details of compactification. We have made use of the amplitudes evaluated near the first resonant pole to report on the discovery potential at the LHC for the first Regge excitations of the quark and gluon. The precise predictions for the branching fraction of two different topologies (dijet and $\gamma+$ jet) can be used as a powerful discriminator of low mass string excitations from other beyond SM scenarios. We have long imagined strings to be minuscule objects which could only be experimentally observed in the far-distant future. It is conceivable that this future has already arrived.

\section{Appendices}

\section{A. TeV-Scale Strings and Large Extra Dimensions}

For an illustration, consider type II string theory compactified on a six-dimensional torus $T^{6}$, which includes a D $p$-brane wrapped around $p-3$ dimensions of $T^{6}$ with the remaining 
dimensions along our familiar (uncompactified) three spatial dimensions. We denote the radii of the internal longitudinal directions (of the D $p$-brane) by $R_{i}^{\|}, i=1, \ldots, p-3$ and the radii of the transverse directions by $R_{j}^{\perp}, j=1, \ldots, 9-p$, see Figure 8 .

The 4-dimensional Planck mass $M_{\mathrm{Pl}} \sim 10^{19} \mathrm{GeV}$, which is related to the string mass scale $M_{S}$ by

$$
M_{\mathrm{Pl}}^{2}=\frac{8}{g_{s}^{2}} M_{s}^{8} \frac{V_{6}}{(2 \pi)^{6}}
$$

determines the strength of the gravitational interactions [106]. Here,

$$
V_{6}=(2 \pi)^{6} \prod_{i=1}^{p-3} R_{i}^{\|} \prod_{j=1}^{9-p} R_{j}^{\perp}
$$

is the volume of $T^{6}$ and $g_{s}$ is the string coupling. It follows that the string scale can be chosen hierarchically smaller than the Planck mass at the expense of introducing $n=9-p$ large transverse dimensions felt only by gravity, while keeping the string coupling small. For example, for a string mass scale $M_{s} \approx \mathcal{O}(1 \mathrm{TeV})$ the volume of the internal space needs to be as large as $V_{6} M_{s}^{6} \approx \mathcal{O}\left(10^{32}\right)$.

On the other hand, the strength of coupling of the gauge theory living on the D-brane world volume is not enhanced as long as $R_{i}^{\|} \sim M_{s}^{-1}$ remain small,

$$
\frac{1}{g^{2}}=\frac{1}{2 \pi g_{s}} M_{s}^{p-3} \prod_{i=1}^{p-3} R_{i}^{\|} .
$$

The weakness of the effective 4-dimensional gravity compared to gauge interactions (ratio of $\left.\langle H\rangle / M_{\mathrm{Pl}}\right)$ is then attributed to the largeness of the transverse space radii $R_{i}^{\perp} \sim 10^{32} l_{s}$ compared to the string length $l_{s}=M_{s}^{-1}$.

A distinct property of these D-brane models is that gravity becomes effectively $(4+n)$ dimensional with a strength comparable to those of gauge interactions at the string scale [131]. Equation (A.1) can be understood as a consequence of the $(4+n)$-dimensional Gauss law for gravity, with

$$
M_{*}^{2+n} \sim \frac{1}{g_{s}^{2}} M_{s}^{2+n}
$$

as the effective scale of gravity in $4+n$ dimensions. Taking $M_{s} \sim 1 \mathrm{TeV}$, one finds a size for the extra dimensions $R^{\perp} \approx 10^{30 / n-19} \mathrm{~m}$. This relation immediately suggests that $n=1$ is ruled out, because $R^{\perp} \sim 10^{11} \mathrm{~m}$ and the gravitational interaction would thus be modified at the scale of our solar system. However, already for $n=2$ one obtains $R^{\perp} \sim 1 \mathrm{~mm}$. This is just the scale where our present day experimental knowledge about gravity ends [132-134].

It is important to note that the mass scale $M_{\mathrm{BH}} \sim M_{s} / g_{s}^{2}$, which corresponds to the onset of black hole production, cannot be probed at the LHC [127]. To be specific, we choose 


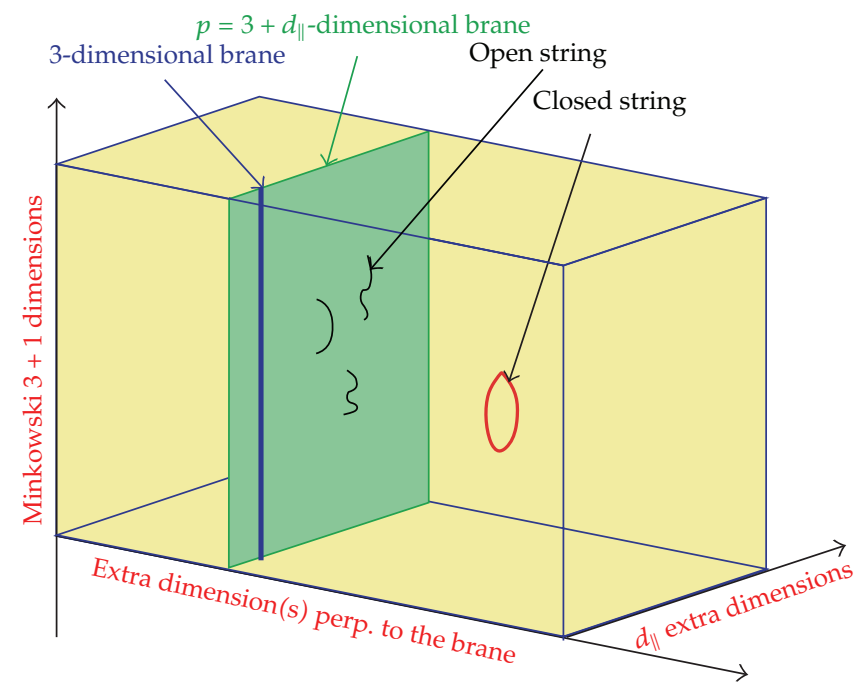

Figure 8: D-brane setup with $d_{\|}$parallel and $d_{\perp}$ transverse internal directions. From [130].

$g_{s}=0.1$ and then we obtain $M_{\mathrm{BH}} \sim 100 M_{s}$. It is also noteworthy that TeV-scale string Dbrane compactifications naturally and unavoidably give rise to "the incredible bulk" which characterizes the recently proposed dynamical dark matter framework $[135,136]$.

\section{B. Pole Residues of the Veneziano form Factor}

Consider the product of Gamma functions

$$
\Gamma(n) \Gamma(1-n)=\frac{\pi}{\sin (n \pi)} .
$$

In the limit $1-n=\epsilon \ll 1, \sin (n \pi)=\sin (\pi-\pi \epsilon)=\sin (\pi)-\pi \epsilon \cos (\pi)=\pi \epsilon$, and so

$$
\Gamma(1-\epsilon) \Gamma(\epsilon)=\frac{\pi}{\pi \epsilon}=\frac{1}{\epsilon},
$$

which in turn leads to

$$
\lim _{n \rightarrow 1} \Gamma(1-n)=\frac{1}{1-n} .
$$

Therefore, in the limit of $s / M_{s}^{2} \rightarrow 1$,

$$
\begin{gathered}
\mu(s, t, u) \longrightarrow \frac{\Gamma\left(1-u / M_{s}^{2}\right)}{\Gamma\left(1+t / M_{s}^{2}\right)} \frac{1}{\left(1-s / M_{s}^{2}\right)}=\frac{\Gamma\left(2+t / M_{s}^{2}\right)}{\Gamma\left(1+t / M_{s}^{2}\right)} \frac{1}{1-s / M_{s}^{2}}=\frac{1+t / M_{s}^{2}}{1+s / M_{s}^{2}} \\
\mu(s, u, t) \longrightarrow \frac{\Gamma\left(1-t / M_{s}^{2}\right)}{\Gamma\left(1+u / M_{s}^{2}\right)} \frac{1}{\left(1-s / M_{s}^{2}\right)}=\frac{\Gamma\left(2+u / M_{s}^{2}\right)}{\Gamma\left(1+u / M_{s}^{2}\right)} \frac{1}{1-s / M_{s}^{2}}=\frac{1+u / M_{s}^{2}}{1+s / M_{s}^{2}}
\end{gathered}
$$


Recall we are working on the physical region (where $t$ and $u$ are negatives) and so the second term in $\mu(s, u, t)$ or $\mu(s, t, u)$ does not develop Regge poles. We can now expand the string squared amplitude,

$$
\begin{aligned}
|\mathcal{M}(g g \longrightarrow \gamma g)|^{2} \propto & \left|\frac{s}{u} \mu(s, t, u)+\frac{s}{t} \mu(s, u, t)\right|^{2}+\left|\frac{t}{u} \mu(s, t, u)+\frac{t}{s} \mu(t, u, s)\right|^{2} \\
& +\left|\frac{u}{s} \mu(u, t, s)+\frac{u}{t} \mu(u, s, t)\right|^{2}
\end{aligned}
$$

near the pole yielding

$$
\begin{aligned}
|\mathcal{M}(g g \longrightarrow r g)|^{2} \propto & \left|\frac{M_{s}^{2}}{u} \frac{\Gamma\left(1-u / M_{s}^{2}\right)}{\Gamma\left(1+t / M_{s}^{2}\right)}+\frac{M_{s}^{2}}{t} \frac{\Gamma\left(1-t / M_{s}^{2}\right)}{\Gamma\left(1+u / M_{s}^{2}\right)}\right|^{2} \frac{1}{\left(s-M_{s}^{2}\right)^{2}} \\
& +\left|-\frac{t}{u} \frac{\Gamma\left(1-u / M_{s}^{2}\right)}{\Gamma\left(1+t / M_{s}^{2}\right)}+\frac{t}{M_{s}^{2}}\left[\frac{\Gamma\left(1-t / M_{s}^{2}\right)}{\Gamma\left(1+u / M_{s}^{2}\right)}-\frac{\Gamma\left(1-u / M_{s}^{2}\right)}{\Gamma\left(1+t / M_{s}^{2}\right)}\right]\right|^{2} \frac{1}{\left(s-M_{s}^{2}\right)^{2}} \\
& +\left|\frac{u}{M_{s}^{2}}\left[\frac{\Gamma\left(1-u / M_{s}^{2}\right)}{\Gamma\left(1+t / M_{s}^{2}\right)}-\frac{\Gamma\left(1-t / M_{s}^{2}\right)}{\Gamma\left(1+u / M_{s}^{2}\right)}\right]-\frac{u}{t} \frac{\Gamma\left(1-t / M_{s}^{2}\right)}{\Gamma\left(1+u / M_{s}^{2}\right)}\right|^{2} \frac{1}{\left(s-M_{s}^{2}\right)^{2}} .
\end{aligned}
$$

Equivalently,

$$
\begin{aligned}
|\mathcal{M}(g g \longrightarrow \gamma g)|^{2} \propto\{ & \left|\frac{M_{s}^{2}}{u} A+\frac{M_{s}^{2}}{t} B\right|^{2}+\left|-\frac{t}{u} A+\frac{t}{M_{s}^{2}}(B-A)\right|^{2} \\
& \left.+\left|\frac{u}{M_{s}^{2}}(A-B)-\frac{u}{t} B\right|^{2}\right\} \frac{1}{\left(s-M_{s}^{2}\right)^{2}},
\end{aligned}
$$

where

$$
\begin{aligned}
& A=\frac{\Gamma\left(1-u / M_{s}^{2}\right)}{\Gamma\left(1+t / M_{s}^{2}\right)}=1+\frac{t}{M_{s}^{2}}=-\frac{u}{M_{s}^{2}}, \\
& B=\frac{\Gamma\left(1-t / M_{s}^{2}\right)}{\Gamma\left(1+u / M_{s}^{2}\right)}=1+\frac{u}{M_{s}^{2}}=-\frac{t}{M_{s}^{2}}
\end{aligned}
$$


are obtained from (B.4). Then, (B.7) becomes

$$
\begin{aligned}
|\mathcal{M}(g g \longrightarrow \gamma g)|^{2} & \propto\left\{4 M_{s}^{4}+\left|t+\frac{t}{M_{s}^{2}}(-t+u)\right|^{2}+\left|u+\frac{u}{M_{s}^{2}}(-u+t)\right|^{2}\right\} \frac{1}{\left(s-M_{s}^{2}\right)^{2}} \\
& \propto\left[4 M_{s}^{4}+\frac{4 t^{4}+4 u^{4}}{M_{s}^{4}}\right] \frac{1}{\left(s-M_{s}^{2}\right)^{2}},
\end{aligned}
$$

where we have used the Mandelstam relation: $u=-M_{s}^{2}-t$.

\section{Acknowledgments}

The author is thankful to Ignatios Antoniadis, Vic Feng, Haim Goldberg, Xing Huang, Dieter Lüst, Satoshi Nawata, Stephan Stieberger, and Tom Taylor for fruitful and enjoyable collaborations. L. A. Anchordoqui is partially supported by the U.S. National Science Foundation (NSF) under Grant PHY-0757598 and CAREER Award PHY-1053663. Any opinions, findings, and conclusions or recommendations expressed in this material are those of the author and do not necessarily reflect the views of the NSF.

\section{References}

[1] K. Nakamura and Particle Data Group, "Review of particle physics," Journal of Physics G, vol. 37, no. 7, article 075021, 2010.

[2] F. Halzen and A. D. Martin, Quarks and leptons: an introductory course in modern particle physics, John Wiley \& Sons, New York, NY, USA, 1984.

[3] M. C. Gonzalez-Garcia and M. Maltoni, "Phenomenology with massive neutrinos," Physics Reports, vol. 460, no. 1-3, pp. 1-129, 2008.

[4] J. Wess and J. Bagger, Supersymmetry and Supergravity, Princeton University Press, Princeton, NJ, USA, 2nd edition, 1992.

[5] L. A. Anchordoqui, I. Antoniadis, H. Goldberg, X. Huang, D. Lust, and T. R. Taylor, "Z'-gauge bosons as harbingers of low mass strings." In press. http://arxiv.org/abs/1107.4309.

[6] L. E. Ibáñez, F. Marchesano, and R. Rabadán, "Getting just the standard model at intersecting branes," High Energy Physics. In press. http:/ /arxiv.org/abs/hep-th/0105155.

[7] D. Cremades, L. E. Ibáñez, and F. Marchesano, "Yukawa couplings in intersecting D-brane models," Journal of High Energy Physics, vol. 7, no. 7, pp. 899-945, 2003.

[8] F. G. Marchesano Buznego, "Intersecting D-brane models," Journal of High Energy Physics. In press. http:/ /arxiv.org/abs/hepth/0307252.

[9] R. D. Peccei and H. R. Quinn, "CP conservation in the presence of pseudoparticles," Physical Review Letters, vol. 38, no. 25, pp. 1440-1443, 1977.

[10] S. Weinberg, "A new light boson?" Physical Review Letters, vol. 40, no. 4, pp. 223-226, 1978.

[11] F. Wilczek, "Problem of strong P and T invariance in the presence of instantons," Physical Review Letters, vol. 40, no. 5, pp. 279-282, 1978.

[12] I. Antoniadis, E. Kiritsis, and T. N. Tomaras, "A D-brane alternative to unification," Physics Letters B, vol. 486, no. 1-2, pp. 186-193, 2000.

[13] P. Anastasopoulos, T. Dijkstra, E. Kiritsis, and B. Schellekens, "Orientifolds, hypercharge embeddings and the standard model," Nuclear Physics B, vol. 759, no. 1-2, pp. 83-146, 2006.

[14] F. Del Aguila, G. D. Coughlan, and M. Quirós, “Gauge coupling renormalisation with several U(1) factors," Nuclear Physics B, vol. 307, no. 3, pp. 633-648, 1988.

[15] F. Del Aguila, J. A. González, and M. Quirós, "Renormalization group analysis of extended electroweak models from the heterotic string," Nuclear Physics B, vol. 312, no. 3, pp. 571-632, 1989.

[16] H. Goldberg. In press.

[17] D. Ghilencea, L. E. Ibáñez, N. Irges, and F. Quevedo, “TeV-scale Z' bosons from D-branes," Journal of High Energy Physics, vol. 6, no. 8, pp. 303-347, 2002. 
[18] T. Aaltonen, B. Álvarez González, S. Amerio et al., "Invariant mass distribution of jet pairs produced in association with a $\mathrm{W}$ Boson in $\mathrm{p} \overline{\mathrm{p}}$ Collisions at $\sqrt{ } \mathrm{s}=1.96 \mathrm{TeV}$, " Physical Review Letters, vol. 106, no. 17, article 171801, 2011.

[19] V. Cavaliere, Measurement of ww+wz production cross section and study of the dijet mass spectrum in the Inu + jets final state at CDF, Ph.D. thesis, 2010, FERMILAB-THESIS-2010-51.

[20] G. Punzi, in Proceedings of the 23th Recontres de Blois, Blois France, May 2011.

[21] A. Annovi, P. Catastini, V. Cavaliere, and L. Ristori, http://www-cdf.fnal.gov/physics/ewk/2011/ wjj/.

[22] V. M. Abazov, B. Abbott, B. S. Acharya et al., "Bounds on an anomalous dijet resonance in W+jets production in $\mathrm{p} \overline{\mathrm{p}}$ collisions at $\sqrt{\mathrm{s}}=1.96 \mathrm{TeV}$, " Physical Review Letters, vol. 107, no. 1, 2011.

[23] E. J. Eichten, K. Lane, and A. Martin, "Technicolor explanation for the CDF wij excess," Physical Review Letters, vol. 106, no. 25, 2011.

[24] C. Kilic and S. Thomas, "Signatures of resonant super-partner production with charged-current decays," Journal of High Energy Physics. In press. http:/ / arxiv.org/abs/1104.1002.

[25] A. E. Nelson, T. Okui, and T. S. Roy, "A unified, flavor symmetric explanation for the t-tbar asymmetry and Wjj excess at CDF," Journal of High Energy Physics. In press. http:/ / arxiv.org/abs/1104.2030.

[26] B. A. Dobrescu and G. Z. Krnjaic, "Weak-triplet, color-octet scalars and the CDF dijet excess," Journal of High Energy Physics. In press. http://arxiv.org/abs/1104.2893.

[27] S. Jung, A. Pierce, and J. D. Wells, "Top quark asymmetry and dijet resonances," Journal of High Energy Physics. In press. http://arxiv.org/abs/1104.3139.

[28] M. Buckley, P. Fileviez Pérez, D. Hooper, and E. Neil, “Dark forces at the Tevatron," Physics Letters B, vol. 702, no. 4, pp. 256-259, 2011.

[29] T. Plehn and M. Takeuchi, "W+jets at CDF: evidence for top quarks," Journal of Physics G, vol. 38, no. 9, 2011.

[30] Q.-H. Cao, M. Carena, S. Gori et al., "W plus two jets from a quasi-inert Higgs doublet," Journal of High Energy Physics, vol. 2011, no. 8, 2011.

[31] J. Fan, D. Krohn, P. Langacker, and I. Yavin, “A Higgsophilic s-channel Z' and the CDF W+2J Anomaly," Journal of High Energy Physics. In press. http:/ / arxiv.org/abs/1106.1682.

[32] J. L. Evans, B. Feldstein, W. Klemm, H. Murayama, and T. T. Yanagida, "Hermitian flavor violation," Journal of High Energy Physics. In press. http:/ / arxiv.org/abs/1106.1734.

[33] R. Harnik, G. D. Kribs, and A. Martin, "Quirks at the tevatron and beyond," Journal of High Energy Physics. In press. http://arxiv.org/abs/1106.2569.

[34] J. F. Gunion, "A two-Higgs-doublet interpretation of a small Tevatron Wjj excess," Journal of High Energy Physics. In press. http://arxiv.org/abs/1106.3308.

[35] M. R. Buckley, D. Hooper, J. Kopp, and E. Neil, “Light Z' bosons at the Tevatron," Physical Review D, vol. 83, no. 11, article 115013, 2011.

[36] F. Yu, "Z' model for the CDF dijet anomaly," Physical Review D, vol. 83, no. 9, 2011.

[37] K. Cheung and J. Song, "Baryonic Z' explanation for the cdf Wij excess," Physical Review Letters, vol. 106, no. 21, 2011.

[38] L. A. Anchordoqui, H. Goldberg, X. Huang, D. Lüst, and T. R. Taylor, "Stringy origin of Tevatron Wjj anomaly," Physics Letters B, vol. 701, no. 2, pp. 224-228, 2011.

[39] P. Ko, Y. Omura, and C. Yu, "Dijet resonance from leptophobic Z' and light baryonic cold dark matter," Journal of High Energy Physics. In press. http:/ / arxiv.org/abs/1104.4066.

[40] P. J. Fox, J. Liu, D. Tucker-Smith, and N. Weiner, "An Effective Z'," Journal of High Energy Physics. In press. http://arxiv.org/abs/1104.4127.

[41] Z. Liu, P. Nath, and G. Peim, "An explanation of the CDF dijet anomaly within a U(1)X Stueckelberg extension," Physics Letters B, vol. 701, no. 5, pp. 601-604, 2011.

[42] M. R. Buckley, D. Hooper, and J. L. Rosner, "A Leptophobic Z' And dark matter from grand unification," Journal of High Energy Physics. In press. http:/ /arxiv.org/abs/1106.3583.

[43] A. E. Faraggi and V. M. Mehta, "Leptophobic Z' in heterotic-string derived models," Journal of High Energy Physics. In press. http://arxiv.org/abs/1106.5422.

[44] K. Cheung and J. Song, "Golden mode for a baryonic $Z$ ' boson at hadronic colliders: $\mathrm{Pp} / \mathrm{p} \overline{\mathrm{p}} \rightarrow$

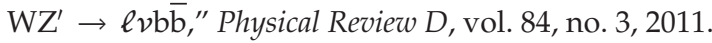

[45] D. Acosta, J. Adelman, T. Affolder et al., "Search for new physics using high-mass tau pairs from $1.96 \mathrm{TeV}$ p $\overline{\mathrm{p}}$ collisions," Physical Review Letters, vol. 95, no. 13, 2005.

[46] T. Aaltonen, A. Abulencia, J. Adelman et al., "Search for new physics in high-mass electron-positron events in p $\bar{p}$ collisions at s=1.96 TeV," Physical Review Letters, vol. 99, no. 17, 2007. 
[47] T. Aaltonen, J. Adelman, T. Akimoto et al., "Search for high-mass resonances decaying to dimuons at CDF," Physical Review Letters, vol. 102, no. 9, 2009.

[48] R. Barate, D. Decamp, P. Ghez et al., "Study of fermion pair production in $\mathrm{e}^{+} \mathrm{e}^{-}$collisions at 130183 GeV," European Physical Journal C, vol. 12, no. 2, pp. 183-207, 2000.

[49] P. Abreu, W. Adam, T. Adye et al., "Measurement and interpretation of fermion-pair production at LEP energies of 183 and 189 GeV," Physics Letters B, vol. 485, no. 1-3, pp. 45-61, 2000.

[50] J. Abdallah, P. Abreu, W. Adam et al., "Measurement and interpretation of fermion-pair production at LEP energies above the Z resonance," European Physical Journal C, vol. 45, no. 3, pp. 589-632, 2006.

[51] Y. Umeda, G. -C. Cho, and K. Hagiwara, "Constraints on leptophobic Z' models from electroweak experiments," Physical Review D, vol. 58, no. 11, article 1150081, 1998.

[52] F. Abe, M. G. Albrow, S. R. Amendolia et al., "Search for new particles decaying to dijets in $p \bar{p}$ collisions at s=1.8 TeV," Physical Review Letters, vol. 74, no. 18, pp. 3538-3543, 1995.

[53] F. Abe, H. Akimoto, A. Akopian et al., "Search for new particles decaying to dijets at CDF," Physical Review D, vol. 55, no. 9, pp. R5263-R5268, 1997.

[54] B. Abbott, M. Abolins, V. Abramov et al., "Dijet mass spectrum and a search for quark compositeness in $\bar{p}$ p collisions at $\sqrt{ } \mathrm{s}=1.8 \mathrm{TeV}$," Physical Review Letters, vol. 82, no. 12, pp. 2457-2462, 1999.

[55] T. Aaltonen, J. Adelman, T. Akimoto et al., "Search for new particles decaying into dijets in protonantiproton collisions at s=1.96 TeV," Physical Review D, vol. 79, no. 11, 2009.

[56] R. Ansari, R. E. Ansorge, D. Autiero et al., "A measurement of two-jet decays of the W and Z bosons at the CERN pbarp-collider," Zeitschrift für Physik C, vol. 49, no. 1, pp. 17-28, 1991.

[57] G. Ambrosini, R. Ansari, D. Autiero et al., "A search for new intermediate vector bosons and excited quarks decaying to two-jets at the CERN p p collider," Nuclear Physics B, vol. 400, no. 1-3, pp. 3-22, 1993.

[58] J. L. Hewett and T. G. Rizzo, "Dissecting the Wjj Anomaly: diagnostic tests of a leptophobic Z'," Journal of High Energy Physics. In press. http://arxiv.org/abs/1106.0294.

[59] M. Williams, C. P. Burgess, A. Maharana, and F. Quevedo, "New constraints (and Motivations) for abelian gauge bosons in the MeV-TeV mass range," Journal of High Energy Physics. In press. http://arxiv.org/abs/1103.4556.

[60] E. Eichten, K. Lane, and A. Martin, "Testing CDF's dijet excess and technicolor at the LHC," Journal of High Energy Physics. In press. http:/ / arxiv.org/abs/1107.4075.

[61] K. Harigaya, R. Sato, and S. Shirai, "LHC Test of CDF Wjj anomaly," Journal of High Energy Physics. In press. http:/ / arxiv.org/abs/1107.5265.

[62] M. R. Buckley, D. Hooper, J. Kopp, A. Martin, and E. T. Neil, "What the Tevatron found?" Journal of High Energy Physics. In press. http:/ / arxiv.org/abs/1107.5799.

[63] J. Pumplin, D. R. Stump, J. Huston, H.-L. Lai, P. Nadolsky, and W. -K. Tung, "New generation of parton distributions with uncertainties from global QCD analysis," Journal of High Energy Physics, vol. 6, no. 7, pp. 325-371, 2002.

[64] Z. Kunszt and D. E. Soper, "Calculation of jet cross sections in hadron collisions at order s3," Physical Review D, vol. 46, no. 1, pp. 192-221, 1992.

[65] S. Chatrchyan, V. Khachatryan, A. M. Sirunyan et al., "Search for resonances in the dijet mass spectrum from 7 TeV pp collisions at CMS," Physics Letters, vol. 704, no. 3, pp. 123-142, 2011.

[66] G. Aad and ATLAS Collaboration, "Search for dilepton resonances in pp collisions at sqrt(s)=7 TeV with the ATLAS detector," Journal of High Energy Physics. In press. http:/ /arxiv.org/abs/1108.1582.

[67] V. Barger, K. Cheung, and P. Langacker, "Baryonic Z' connection of LEP $\mathrm{R}_{b, c}$ data with Tevatron (W, Z, $\gamma$ ) b $\bar{b}$ events," Physics Letters B, vol. 381, no. 1-3, pp. 226-236, 1996.

[68] V. Barger and R. J. N. Phillips, Collider Physics, Addison-Wesley, 1987.

[69] J. Polchinski, "Dirichlet branes and Ramond-Ramond charges," Physical Review Letters, vol. 75, no. 26, pp. 4724-4727, 1995.

[70] J. Polchinski, “TASI lectures on D-Branes," Journal High Energy Physics. In press. http://arxiv.org/ abs/hep-th/9611050.

[71] I. Antoniadis, N. Arkani-Hamed, S. Dimopoulos, and G. Dvali, "New dimensions at a millimeter to a fermi and superstrings at a TeV," Physics Letters B, vol. 436, no. 3-4, pp. 257-263, 1998.

[72] R. Blumenhagen, B. Körs, D. Lüst, and T. Ott, "The standard model from stable intersecting brane world orbifolds," Nuclear Physics B, vol. 616, no. 1-2, pp. 3-33, 2001.

[73] M. Cvetic, G. Shiu, and A. M. Uranga, "Three-family supersymmetric standardlike models from intersecting brane worlds," Physical Review Letters, vol. 87, no. 20, pp. 201801/1-201801/4, 2001.

[74] M. Cvetič, G. Shiu, and A. M. Uranga, "Chiral four-dimensional N=1 supersymmetric type IIA orientifolds from intersecting D6-branes," Nuclear Physics B, vol. 615, no. 1-3, pp. 3-32, 2001. 
[75] I. Antoniadis, E. Kiritsis, and T. Tomaras, "D-brane standard model," Fortschritte der Physik, vol. 49, no. 4-6, pp. 573-580, 2001.

[76] E. Kiritsis and P. Anastasopoulos, "The anomalous magnetic moment of the muon in the D-brane realization of the Standard Model," Journal of High Energy Physics, vol. 6, no. 5, pp. 1291-1312, 2002.

[77] G. Honecker and T. Ott, "Getting just the supersymmetric standard model at intersecting branes on the $\mathrm{Z}_{6}$ orientifold," Physical Review D, vol. 70, no. 12, article 126010, p. 25, 2004.

[78] F. Gmeiner, R. Blumenhagen, G. Honecker, D. Lüst, and T. Weigand, “One in a billion: MSSM-like D-brane statistics," Journal of High Energy Physics, no. 1, pp. 58-87, 2006.

[79] F. Gmeiner and G. Honecker, "Millions of standard models on $\mathrm{Z}_{6}$ ? " Journal of High Energy Physics, vol. 2008, no. 7, p. 052, 2008.

[80] E. Kiritsis, "D-branes in standard model building, gravity and cosmology," Physics Reports, vol. 421, no. 3-4, pp. 105-190, 2005.

[81] R. Blumenhagen, M. Cvetič, P. Langacker, and G. Shiu, "Toward realistic intersecting D-brane models," Annual Review of Nuclear and Particle Science, vol. 55, pp. 71-139, 2005.

[82] R. Blumenhagen, B. Körs, D. Lüst, and S. Stieberger, "Four-dimensional string compactifications with D-branes, orientifolds and fluxes," Physics Reports, vol. 445, no. 1-6, pp. 1-193, 2007.

[83] I. Antoniadis and S. Dimopoulos, "Splitting supersymmetry in string theory," Nuclear Physics B, vol. 715, no. 1-2, pp. 120-140, 2005.

[84] D. Berenstein and S. Pinansky, "Minimal quiver standard model," Physical Review D, vol. 75, no. 9, 2007.

[85] D. Berenstein, R. Martínez, F. Ochoa, and S. Pinansky, " $Z$ ' boson detection in the minimal quiver standard model," Physical Review D, vol. 79, no. 9, 2009.

[86] M. B. Green and J. H. Schwarz, "Anomaly cancellations in supersymmetric D=10 gauge theory and superstring theory," Physics Letters B, vol. 149, no. 1-3, pp. 117-122, 1984.

[87] E. Witten, "Some properties of O(32) superstrings," Physics Letters B, vol. 149, no. 4-5, pp. 351-356, 1984.

[88] M. Dine, N. Seiberg, and E. Witten, "Fayet-Iliopoulos terms in string theory," Nuclear Physics B, vol. 289, no. 3-4, pp. 589-598, 1987.

[89] J. J. Atick, L. J. Dixon, and A. Sen, "String calculation of Fayet-Iliopoulos D-terms in arbitrary supersymmetric compactifications," Nuclear Physics B, vol. 292, no. 1, pp. 109-149, 1987.

[90] W. Lerche, B. E. W. Nilsson, A. N. Schellekens, and N. P. Warner, "Anomaly cancelling terms from the elliptic genus," Nuclear Physics B, vol. 299, no. 1, pp. 91-116, 1988.

[91] I. Antoniadis, E. Kiritsis, and J. Rizos, "Anomalous U(1)'s in type I string vacua," Nuclear Physics B, vol. 637, no. 1-3, pp. 92-118, 2002.

[92] P. Anastasopoulos, "4D anomalous U(1)'s, their masses and their relation to 6D anomalies," Journal of High Energy Physics, vol. 7, no. 8, pp. 107-129, 2003.

[93] I. Antoniadis, E. Kiritsis, J. Rizos, and T. N. Tomaras, "D-branes and the standard model," Nuclear Physics B, vol. 660, no. 1-2, pp. 81-115, 2003.

[94] J. P. Conlon, A. Maharana, and F. Quevedo, "Towards realistic string vacua from branes at singularities," Journal of High Energy Physics, no. 5, p. 109, 2009.

[95] M. Cvetic, J. Halverson, and P. Langacker, "Implications of string constraints for exotic matter and $\mathrm{Z}^{\prime} \mathrm{s}$ beyond the standard model," Journal of High Energy Physics. In press. http:// arxiv.org/abs/1108.5187.

[96] M. Cvetič, J. Halverson, and R. Richter, "Realistic Yukawa structures from orientifold compactifications," Journal of High Energy Physics, no. 12, p. 063, 2009.

[97] S. J. Parke and T. R. Taylor, "Amplitude for n-Gluon Scattering," Physical Review Letters, vol. 56, no. 23, pp. 2459-2460, 1986.

[98] S. Stieberger and T. R. Taylor, “Amplitude for N-gluon superstring scattering," Physical Review Letters, vol. 97, no. 21, article 211601, 2006.

[99] S. Stieberger and T. R. Taylor, "Multigluon scattering in open superstring theory," Physical Review D, vol. 74, no. 12, article 126007, p. 19, 2006.

[100] M. L. Mangano and S. J. Parke, "Multi-parton amplitudes in gauge theories," Physics Report, vol. 200, no. 6, pp. 301-367, 1991.

[101] L. J. Dixon, "Calculating Scattering Amplitudes Efficiently," Journal of High Energy Physics. In press.http://arxiv.org/abs/hep-ph/9601359.

[102] G. Veneziano, "Construction of a crossing-simmetric, Regge-behaved amplitude for linearly rising trajectories," Il Nuovo Cimento A, vol. 57, no. 1, pp. 190-197, 1968. 
[103] L. A. Anchordoqui, H. Goldberg, S. Nawata, and T. R. Taylor, "Jet signals for low mass strings at the large hadron collider," Physical Review Letters, vol. 100, no. 17, 2008.

[104] L. A. Anchordoqui, H. Goldberg, S. Nawata, and T. R. Taylor, “Direct photons as probes of low mass strings at the CERN LHC," Physical Review D, vol. 78, no. 1, 2008.

[105] T. van Ritbergen, A. N. Schellekens, and J. A. M. Vermaseren, "Group theory factors for Feynman diagrams," International Journal of Modern Physics A, vol. 14, no. 1, pp. 41-96, 1999.

[106] D. Lüst, S. Stieberger, and T. R. Taylor, "The LHC string hunter's companion," Nuclear Physics B, vol. 808, no. 1-2, pp. 1-52, 2009.

[107] L. A. Anchordoqui, H. Goldberg, D. Lüst, S. Nawata, S. Stieberger, and T. R. Taylor, “Dijet signals for low mass strings at the large hadron collider," Physical Review Letters, vol. 101, no. 24, 2008.

[108] L. A. Anchordoqui, H. Goldberg, and T. R. Taylor, "Decay widths of lowest massive Regge excitations of open strings," Physics Letters B, vol. 668, no. 5, pp. 373-377, 2008.

[109] Z. Dong, T. Han, M. -X. Huang, and G. Shiu, "Top quarks as a window to string resonances," Journal of High Energy Physics, vol. 2010, no. 9, 2010.

[110] F. Cornet, J. I. Illana, and M. Masip, “TeV strings and the neutrino-nucleon cross section at ultrahigh energies," Physical Review Letters, vol. 86, no. 19, pp. 4235-4238, 2001.

[111] E. Eichten, I. Hinchliffe, K. Lane, and C. Quigg, "Supercollider physics," Reviews of Modern Physics, vol. 56, no. 4, pp. 579-707, 1984

[112] V. Khachatryan, A. M. Sirunyan, A. Tumasyan et al., "Search for dijet resonances in $7 \mathrm{TeV}$ pp collisions at CMS," Physical Review Letters, vol. 105, no. 21, article 211801, 2010.

[113] V. Khachatryan, A. M. Sirunyan, A. Tumasyan, W. Adam, T. Bergauer, and M. Dragicevic, "Search for dijet resonances in $7 \mathrm{TeV}$ pp collisions at CMS," Physical Review Letters, vol. 106, no. 2, article 029902, 2011.

[114] A. Bhatti, B. Bollen, M. Cardaci et al., "CMS search plans and sensitivity to new physics with dijets," Journal of Physics G, vol. 36, no. 1, 2009.

[115] L. Randall and R. Sundrum, "Large mass hierarchy from a small extra dimension," Physical Review Letters, vol. 83, no. 17, pp. 3370-3373, 1999.

[116] B. Hassanain, J. March-Russell, and J. G. Rosa, "On the possibility of light string resonances at the LHC and Tevatron from randall-sundrum throats," Journal of High Energy Physics, vol. 2009, no. 7, 2009.

[117] M. Perelstein and A. Spray, "Tensor reggeons from warped space at the LHC," Journal of High Energy Physics, vol. 2009, 2009.

[118] L. A. Anchordoqui, H. Goldberg, X. Huang, and T. R. Taylor, "LHC phenomenology of lowest massive regge recurrences in the randall-sundrum orbifold," Physical Review D, vol. 82, no. 10, 2010.

[119] M. Perelstein and A. Spray, "Four boosted tops from a regge gluon," Journal of High Energy Physics, vol. 2011, no. 9, 2011.

[120] L. A. Anchordoqui, H. Goldberg, D. LÜst, S. Stieberger, and T. R. Taylor, "String phenomenology at the LHC," Modern Physics Letters A, vol. 24, no. 31, pp. 2481-2490, 2009.

[121] G. L. Bayatian, S. Chatrchyan, G. Hmayakyan et al., "CMS physics technical design report, volume II: physics performance," Journal of Physics G, vol. 34, no. 6, 2007.

[122] W. W. Armstrong, W. Burris, D. M. Gingrich et al., "Technical proposal for a general-purpose p p experiment at the large hadron collider at CERN," Tech. Rep. CERN/LHCC 94-43, 1994.

[123] P. Gupta, B. C. Choudhary, S. Chatterji, and S. Bhattacharya, "Study of direct photon plus jet production in CMS experiment at $\sqrt{ } \mathrm{s}=14 \mathrm{TeV}$," European Physical Journal C, vol. 53, no. 1, pp. 49-58, 2008.

[124] S. Cullen, M. Perelstein, and M. E. Peskin, "TeV strings and collider probes of large extra dimensions," Physical Review D, vol. 62, no. 5, pp. 1-21, 2000.

[125] L. A. Anchordoqui, H. Goldberg, D. Lüst, S. Nawata, S. Stieberger, and T. R. Taylor, "LHC phenomenology for string hunters," Nuclear Physics B, vol. 821, no. 1-2, pp. 181-196, 2009.

[126] B. Abbott, M. Abolins, V. Abramov et al., "Dijet mass spectrum and a search for quark compositeness in $\bar{p}$ p collisions at $\sqrt{ } \mathrm{s}=1.8 \mathrm{TeV}, "$ Physical Review Letters, vol. 82, no. 12, pp. 2457-2462, 1999.

[127] P. Meade and L. Randall, "Black holes and quantum gravity at the LHC," Journal of High Energy Physics, no. 5, p. 003, 2008.

[128] S. Esen and R. Harris, "CMS sensitivity to quark contact interactions using dijets," CMS Note 2006/071.

[129] L. A. Anchordoqui, W.-Z. Feng, H. Goldberg, X. Huang, and T. R. Taylor, "Searching for string resonances in $\mathrm{e}^{+} \mathrm{e}^{-}$and $\gamma \gamma$ collisions," Physical Review D, vol. 83, no. 10, 2011. 
[130] I. Antoniadis, "The physics of extra dimensions," Lecture Notes in Physics, vol. 720, pp. 293-321, 2007.

[131] N. Arkani-Hamed, S. Dimopoulos, and G. Dvali, "The hierarchy problem and new dimensions at a millimeter," Physics Letters B, vol. 429, no. 3-4, pp. 263-272, 1998.

[132] C. D. Hoyle, U. Schmidt, B. R. Heckel et al., "Submillimeter test of the gravitational inverse-square law: a search for "large" extra dimensions," Physical Review Letters, vol. 86, no. 8, pp. 1418-1421, 2001.

[133] C. D. Hoyle, D. J. Kapner, B. R. Heckel et al., "Submillimeter tests of the gravitational inverse-square law," Physical Review D, vol. 70, no. 4, 2004.

[134] D. J. Kapner, T. S. Cook, E. G. Adelberger et al., "Tests of the gravitational inverse-square law below the dark-energy length scale," Physical Review Letters, vol. 98, no. 2, 2007.

[135] K. R. Dienes and B. Thomas, "Dynamical dark matter: I. Theoreticaloverview," Journal of High Energy Physics. In press, http:/ / arxiv.org/abs/1106.4546.

[136] K. R. Dienes and B. Thomas, “Dynamical dark matter: II. An explicit model,” Journal of High Energy Physics. In press, http:/ / arxiv.org/abs/1107.0721. 

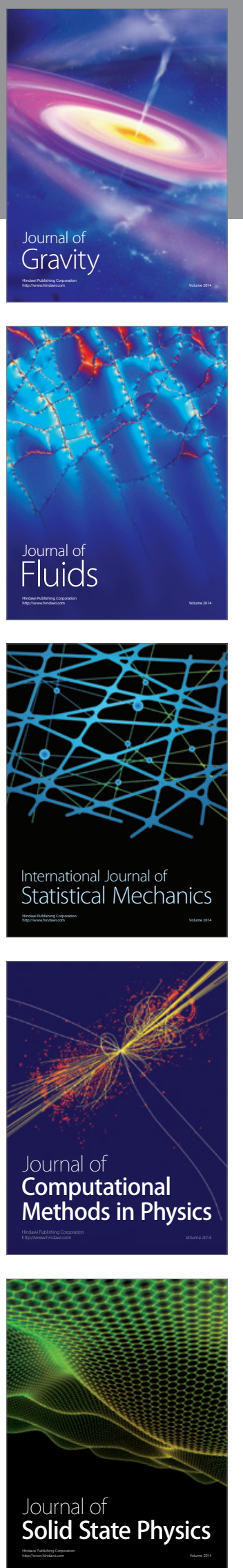

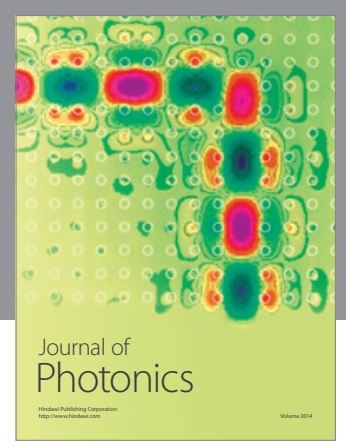

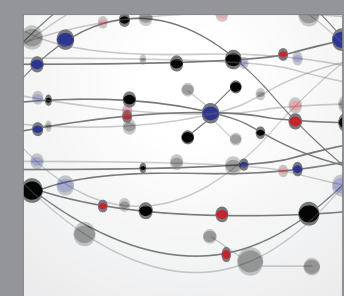

The Scientific World Journal
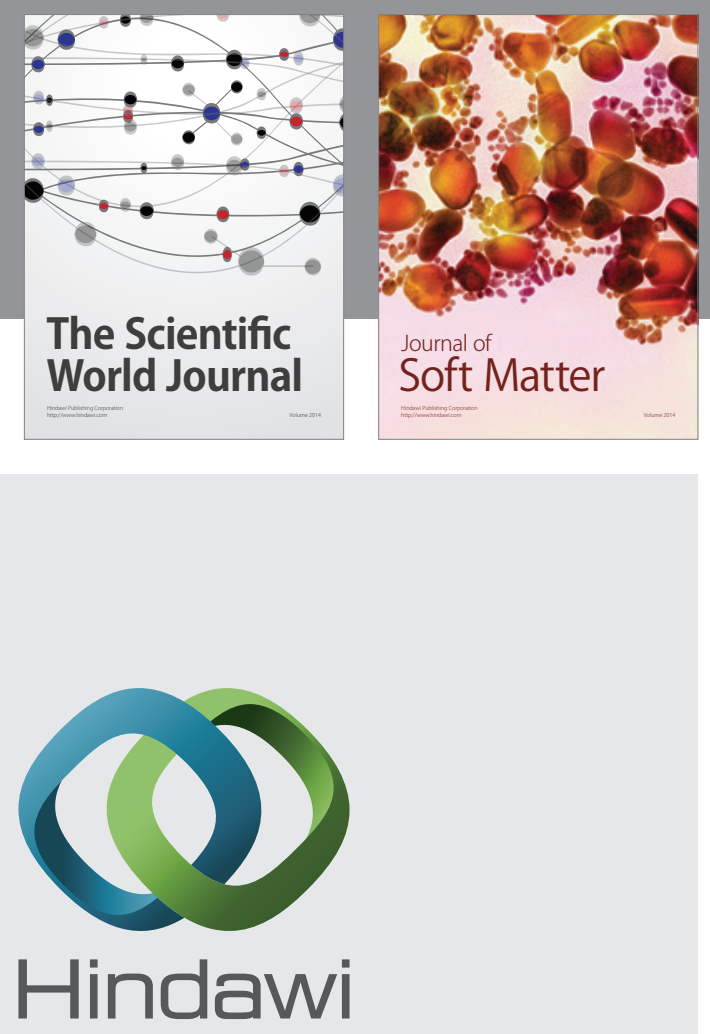

Submit your manuscripts at

http://www.hindawi.com
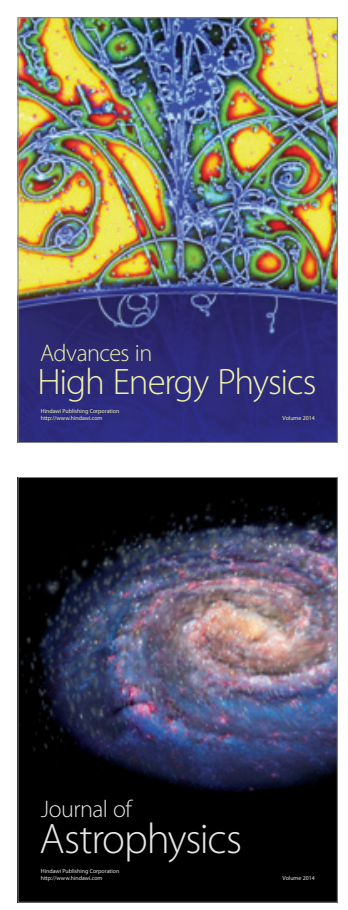
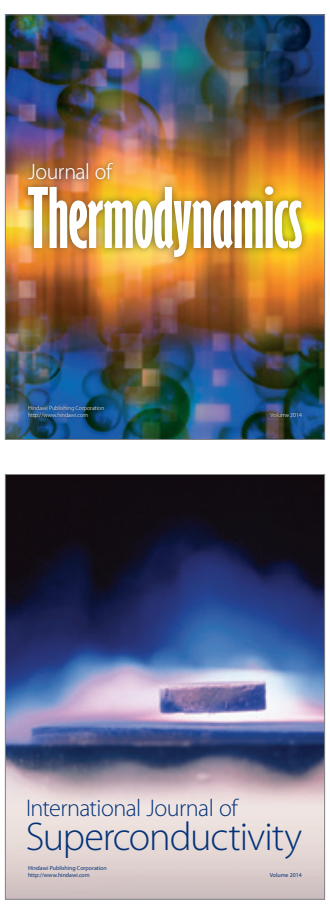
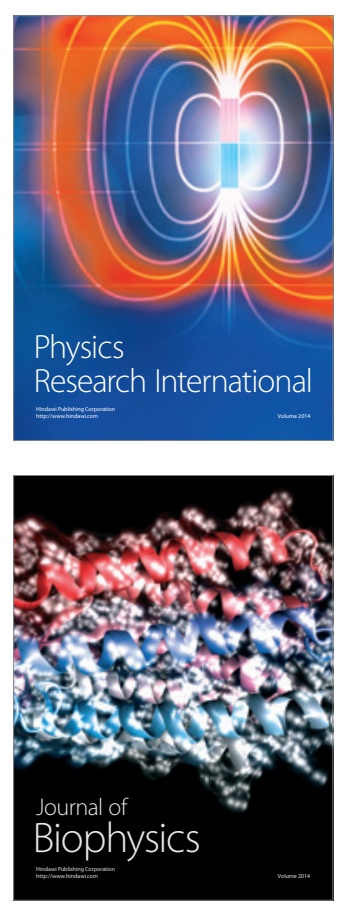
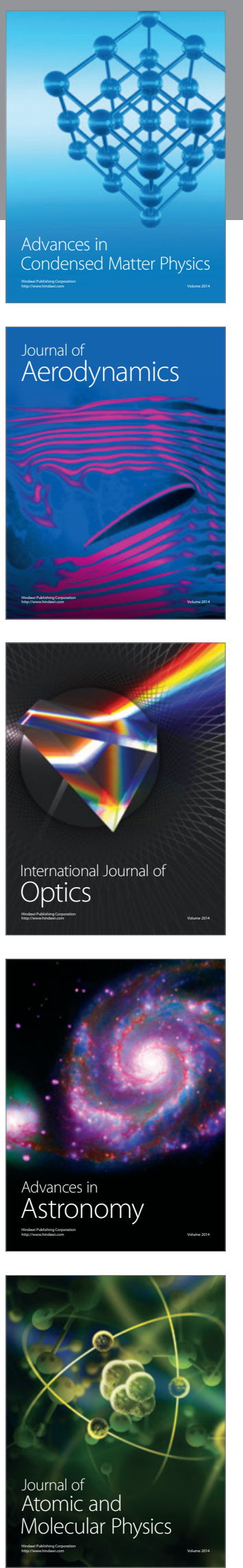